\title{
Is There an Analytic Theory of Automorphic Functions for Complex Algebraic Curves?
}

\author{
Edward FRENKEL
}

Department of Mathematics, University of California, Berkeley, CA 94720, USA

E-mail: frenkel@math.berkeley.edu

Received September 30, 2019, in final form April 27, 2020; Published online May 16, 2020

https://doi.org/10.3842/SIGMA.2020.042

\begin{abstract}
The geometric Langlands correspondence for complex algebraic curves differs from the original Langlands correspondence for number fields in that it is formulated in terms of sheaves rather than functions (in the intermediate case of curves over finite fields, both formulations are possible). In a recent preprint, Robert Langlands made a proposal for developing an analytic theory of automorphic forms on the moduli space of $G$-bundles on a complex algebraic curve. Langlands envisioned these forms as eigenfunctions of some analogues of Hecke operators. In these notes I show that if $G$ is an abelian group then there are well-defined Hecke operators, and I give a complete description of their eigenfunctions and eigenvalues. For non-abelian $G$, Hecke operators involve integration, which presents some difficulties. However, there is an alternative approach to developing an analytic theory of automorphic forms, based on the existence of a large commutative algebra of global differential operators acting on half-densities on the moduli stack of $G$-bundles. This approach (which implements some ideas of Joerg Teschner) is outlined here, as a preview of a joint work with Pavel Etingof and David Kazhdan.
\end{abstract}

Key words: Langlands Program; automorphic function; complex algebraic curve; principal $G$-bundle; Jacobian variety; differential operator; oper

2020 Mathematics Subject Classification: 14D24; 17B67; 22E57

To my teacher Dmitry Borisovich Fuchs on his 80th birthday

\section{Introduction}

1.1. The foundations of the Langlands Program were laid by Robert Langlands in the late 1960s [41]. Originally, these ideas were applied in two realms: that of number fields, i.e., finite extensions of the field $\mathbb{Q}$ of rational numbers, and that of function fields, where by a function field one understands the field of rational functions on a smooth projective curve over a finite field $\mathbb{F}_{q}$. In both cases, the objects of interest are automorphic forms, which are, roughly speaking, functions on the quotient of the form $G(F) \backslash G\left(\mathbb{A}_{F}\right) / K$. Here $F$ is a number field or a function field, $G$ is a reductive algebraic group over $F, \mathbb{A}_{F}$ is the ring of adeles of $F$, and $K$ is a compact subgroup of $G\left(\mathbb{A}_{F}\right)$. There is a family of mutually commuting Hecke operators acting on this space of functions, and one wishes to describe the common eigenfunctions of these operators as well as their eigenvalues. The idea is that those eigenvalues can be packaged as the "Langlands parameters" which can be described in terms of homomorphisms from a group closely related to the Galois group of $F$ to the Langlands dual group ${ }^{L} G$ associated to $G$, and perhaps some additional data.

To be more specific, let $F$ be the field of rational functions on a curve $X$ over $\mathbb{F}_{q}$ and $G=\mathrm{GL}_{n}$. Let us further restrict ourselves to the unramified case, so that $K$ is the maximal compact subgroup $K=\mathrm{GL}_{n}\left(\mathcal{O}_{F}\right)$, where $\mathcal{O}_{F} \subset \mathbb{A}_{F}$ is the ring of integer adeles. In this case,

This paper is a contribution to the Special Issue on Algebra, Topology, and Dynamics in Interaction in honor of Dmitry Fuchs. The full collection is available at https://www.emis.de/journals/SIGMA/Fuchs.html 
a theorem of V. Drinfeld $[12,13,14,15]$ for $n=2$ and L. Lafforgue [40] for $n>2$ states that (if we impose the so-called cuspidality condition and place a restriction on the action of the center of $\left.\mathrm{GL}_{n}\right)$ the Hecke eigenfunctions on $\mathrm{GL}_{n}(F) \backslash \mathrm{GL}_{n}\left(\mathbb{A}_{F}\right) / \mathrm{GL}_{n}\left(\mathcal{O}_{F}\right)$ are in one-to-one correspondence with $n$-dimensional irreducible unramified representations of the Galois group of $F$ (with a matching restriction on its determinant).

1.2. Number fields and function fields for curves over $\mathbb{F}_{q}$ are two "languages" in André Weil's famous trilingual "Rosetta stone" [53], the third language being the theory of algebraic curves over the field $\mathbb{C}$ of complex numbers. Hence it is tempting to build an analogue of the Langlands correspondence in the setting of a complex curve $X$. Such a theory has indeed been developed starting from the mid-1980s, initially by V. Drinfeld [13] and G. Laumon [43] (and relying on the ideas of an earlier work of P. Deligne), then by A. Beilinson and V. Drinfeld [6], and subsequently by many others. See, for example, the surveys [23, 28] for more details. However, this theory, dubbed "geometric Langlands Program", is quite different from the Langlands Program in its original formulation for number fields and function fields.

The most striking difference is that in the geometric theory the vector space of automorphic functions on the double quotient $G(F) \backslash G\left(\mathbb{A}_{F}\right) / K$ is replaced by a (derived) category of sheaves on an algebraic stack whose set of $\mathbb{C}$-points is this quotient. For example, in the unramified case $K=G\left(\mathcal{O}_{F}\right)$, this is the moduli stack $\operatorname{Bun}_{G}$ of principal $G$-bundles on our complex curve $X$. Instead of the Hecke operators of the classical theory, which act on functions, we then have Hecke functors acting on suitable categories of sheaves, and instead of Hecke eigenfunctions we have Hecke eigensheaves.

For example, in the unramified case a Hecke eigensheaf $\mathcal{F}$ is a sheaf on $\operatorname{Bun}_{G}$ (more precisely, an object in the category of $D$-modules on $\operatorname{Bun}_{G}$, or the category of perverse sheaves on $\operatorname{Bun}_{G}$ ) with the property that its images under the Hecke functors are isomorphic to $\mathcal{F}$ itself, tensored with a vector space (this is the categorical analogue of the statement that under the action of the Hecke operators eigenfunctions are multiplied by scalars). Furthermore, since the Hecke functors (just like the Hecke operators acting on functions) are parametrized by closed points of $X$, a Hecke eigensheaf actually yields a family of vector spaces parametrized by points of $X$. We then impose an additional requirement that these vector spaces be stalks of a local system on $X$ for the Langlands dual group ${ }^{L} G$ (taken in the representation of ${ }^{L} G$ corresponding to the Hecke functor under consideration). This neat formulation enables us to directly link Hecke eigensheaves and (equivalence classes of ${ }^{L} G$-local systems on $X$, which are the same as (equivalence classes of) homomorphisms from the fundamental group $\pi_{1}\left(X, p_{0}\right)$ of $X$ to ${ }^{L} G$.

This makes sense from the point of view of Weil's Rosetta stone, because the fundamental group can be seen as a geometric analogue of the unramified quotient of the Galois group of a function field. We note that for $G=\mathrm{GL}_{n}$, in the unramified case, the Hecke eigensheaves have been constructed in [13] for $n=2$ and in [25, 26] for $n>2$. More precisely, the following theorem has been proved: for any irreducible rank $n$ local system $\mathcal{E}$ on $X$, there exists a Hecke eigensheaf on $\mathrm{Bun}_{\mathrm{GL}_{n}}$ whose "eigenvalues" correspond to $\mathcal{E} .{ }^{1}$ Many results of that nature have

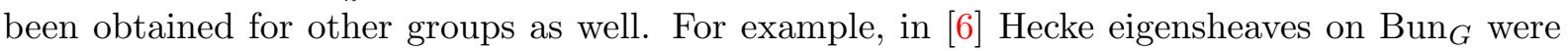
constructed for all ${ }^{L} G$-local systems having the structure of an ${ }^{L} G$-oper (these local systems form a Lagrangian subspace in the moduli of all ${ }^{L} G$-local systems). Furthermore, a more satisfying categorical version of the geometric Langlands correspondence has been proposed by A. Beilinson and V. Drinfeld and developed further in the works of D. Arinkin and D. Gaitsgory [2, 27] (see [28] for a survey).

To summarize, the salient difference between the original formulation of the Langlands Program (for number fields and function fields of curves over $\mathbb{F}_{q}$ ) and the geometric formulation is that the former is concerned with functions and the latter is concerned with sheaves. What makes this geometric formulation appealing is that in the intermediate case - that of curves

\footnotetext{
${ }^{1}$ Furthermore, these Hecke eigensheaves are irreducible on each connected component of $\mathrm{Bun}_{\mathrm{GL}_{n}}$.
} 
over $\mathbb{F}_{q}$ - which serves as a kind of a bridge in the Rosetta stone between the number field case and the case of curves over $\mathbb{C}$, both function-theoretic and sheaf-theoretic formulations make sense. Moreover, it is quite common that the same geometric construction works for curves over $\mathbb{F}_{q}$ and $\mathbb{C}$. For example, essentially the same construction produces Hecke eigensheaves on Bun $_{\mathrm{GL}_{n}}$ for an irreducible rank $n$ local system on a curve over $\mathbb{F}_{q}$ and over $\mathbb{C}$ $[13,25,26] .^{2}$

Furthermore, in the realm of curves over $\mathbb{F}_{q}$, the function-theoretic and sheaf-theoretic formulations are connected to each other by Alexander Grothendieck's "functions-sheaves dictionary". This dictionary assigns to a ( $\ell$-adic) sheaf $\mathcal{F}$ on a variety (or an algebraic stack) $V$ over $\mathbb{F}_{q}$, a function on the set of closed points of $V$ whose value at a given closed point $v$ is the alternating sum of the traces of the Frobenius (a generator of the Galois group of the residue field of $v$ ) on the stalk cohomologies of $\mathcal{F}$ at $v$ (see [44, Section 1.2] or [23, Section 3.3], for details). Thus, for curves over $\mathbb{F}_{q}$ the geometric formulation of the Langlands Program may be viewed as a refinement of the original formulation: the goal is to produce, for each ${ }^{L} G$-local system on $X$, the corresponding Hecke eigensheaf on $\mathrm{Bun}_{G}$, but at the end of the day we can always go back to the more familiar Hecke eigenfunctions by taking the traces of the Frobenius on the stalks of the Hecke eigensheaf at the $\mathbb{F}_{q}$-points of $\operatorname{Bun}_{G}$. Thus, the function-theoretic and the sheaf-theoretic formulations go hand-in-hand for curves over $\mathbb{F}_{q}$.

1.3. In the case of curves over $\mathbb{C}$ there is no Frobenius, and hence no direct way to get functions out of Hecke eigensheaves on $\operatorname{Bun}_{G}$. However, since a Hecke eigensheaf is a $D$-module on $\mathrm{Bun}_{G}$, we could view its sections as analogues of automorphic functions of the analytic theory. The problem is that for non-abelian $G$, these $D$-modules - and hence their sections - are known to have complicated singularities and monodromies. Outside of the singularity locus, a Hecke eigensheaf is a holomorphic vector bundle with a holomorphic flat connection, but its horizontal sections have non-trivial monodromies along the closed paths going around various components of the singularity locus (and in general there are non-trivial monodromies along other closed paths as well). So instead of functions we get multi-valued sections of a vector bundle. On top of that, in the non-abelian case the rank of this vector bundle grows exponentially as a function of the genus of $X$, and furthermore, the components of the singularity locus have a rather complicated structure. Therefore in the non-abelian case, as the genus of $X$ grows, it becomes increasingly difficult to study these horizontal sections. For this reason, it is the $D$-modules themselves, rather than their sections, that are traditionally viewed as more meaningful objects of study, and that's why in the geometric formulation of the Langlands Program for curves over $\mathbb{C}$, we focus on these $D$-modules rather than their multi-valued sections. Thus, the geometric theory in the case of complex curves becomes inherently sheaf-theoretic.

1.4. In a recent preprint [42], Robert Langlands made a proposal for developing an analytic theory of automorphic functions for complex algebraic curves. He mostly considered the case that $X$ is an elliptic curve and $G$ is $\mathrm{GL}_{1}$ or $\mathrm{GL}_{2}$. His proposal can be summarized as follows: (1) He assumed that one can define a commutative algebra of Hecke operators acting on a particular space of $L^{2}$ functions on $\operatorname{Bun}_{G}$ (he only gave a definition of these when $X$ is an elliptic curve and $G=\mathrm{GL}_{2}$ ). (2) He assumed that the Satake isomorphism of the theory over $\mathbb{F}_{q}$ would also hold over $\mathbb{C}$ and that each point $\sigma$ in the joint spectrum of these Hecke operators would give rise to a function $f_{\sigma}$ on the curve $X$ with values in the space of semi-simple conjugacy classes of the maximal compact subgroup ${ }^{L} G_{c}$ of ${ }^{L} G$. (3) He proposed that each function $f_{\sigma}$ could be expressed in terms of the holonomies of a Yang-Mills connection $\nabla_{\sigma}$ on an ${ }^{L} G_{c^{-}}$-bundle on $X$. (4) Atiyah and Bott have shown in [4] that to a Yang-Mills connection $\nabla$ one can associate a homomorphism $\rho(\nabla)$ from a central extension $\widehat{\pi}_{1}(X)$ of the fundamental group $\pi_{1}(X)$ of $X$

\footnotetext{
${ }^{2}$ The term "local system" has different meanings in the two cases: it is an $\ell$-adic sheaf in the first case and a bundle with a flat connection in the second case, but what we do with these local systems to construct Hecke eigensheaves (in the appropriate categories of sheaves) is essentially the same in both cases.
} 
to ${ }^{L} G_{c}$. Langlands proposed that the resulting map $\sigma \mapsto \rho\left(\nabla_{\sigma}\right)$ would give rise to a bijection between the spectrum of the Hecke operators and the set of equivalence classes of homomorphisms $\widehat{\pi}_{1}(X) \rightarrow{ }^{L} G_{c}$ satisfying a certain finiteness condition.

1.5. In this paper, I discuss this proposal. Consider first the case of $\mathrm{GL}_{1}$.

In this case, the Picard variety of a complex curve $X$ plays the role of $\mathrm{Bun}_{\mathrm{GL}_{1}}$ (see Section 2.1). It carries a natural integration measure using which one can define the Hilbert space of $L^{2}$ functions. The Hecke operators are rather simple in the case of $\mathrm{GL}_{1}$ (as well as an arbitrary torus): they are pull-backs of functions under natural maps. Therefore no integration is needed to define an action of the commutative algebra of Hecke operators on this Hilbert space. The question of finding their eigenfunctions and eigenvalues is well-posed.

I give a complete answer to this question in Section 2: first for elliptic curves in Sections 2.1 and 2.2 and then for curves of an arbitrary genus in Section 2.4. In Section 2.5, I generalize these results to the case of an arbitrary torus $T$ instead of $\mathrm{GL}_{1}$. In particular, I show that Hecke eigenfunctions are labeled by $H^{1}\left(X, \Lambda^{*}(T)\right)$, the first cohomology group of $X$ with coefficients in the lattice of cocharacters of $T$, and give an explicit formula for the corresponding eigenvalues. The construction uses the Abel-Jacobi map.

The results presented in Section 2 agree with parts (1), (2), and (3) of Langlands' proposal in the case of $\mathrm{GL}_{1}$. However, the results of Section 2 are not in agreement with part (4) of the proposal. Indeed, each point $\sigma$ in the spectrum of the Hecke operators in the case of $\mathrm{GL}_{1}$ gives rise to a function $f_{\sigma}$ on $X$ with values in $U(1) \subset \mathbb{C}^{\times}$and it is possible to write this function $f_{\sigma}$ as the holonomy of a flat unitary connection $\nabla_{\sigma}$ on a line bundle on $X$. This is shown in Section 2.3 for elliptic curves and in Section 2.4 for general curves. However, and this is a key point, each of these connections necessarily gives rises to the trivial monodromy representation of the fundamental group $\pi_{1}(X)$. Indeed, by construction, $f_{\sigma}$ is a single-valued function on $X$, and it is a horizontal section of the connection $\nabla_{\sigma}$. Therefore the connection $\nabla_{\sigma}$ has trivial monodromy. Thus, the map in part (4) sends each $\sigma$ to the trivial representation of $\pi_{1}(X)$.

1.6. Now consider the case of $\mathrm{GL}_{2}$. Unlike the abelian case, in order to define Hecke operators for non-abelian groups, one cannot avoid integration. Therefore one needs to define the pertinent integration measures. In the classical setting, over $\mathbb{F}_{q}$, the group $G\left(\mathbb{F}_{q}((t))\right)$ is locally compact and therefore carries a Haar measure. Using this Haar measure, one then defines the measures of integration pertinent to the Hecke operators. In contrast, the group $G(\mathbb{C}((t)))$ is not locally compact, and therefore it does not carry a Haar measure, which is only defined for locally compact groups. Therefore, the standard definition of the measure for curves over $\mathbb{F}_{q}$ does not directly generalize to the case of curves over $\mathbb{C}$, as explained in Section 3.

In [42] an attempt is made to explicitly define Hecke operators acting on a particular version of an $L^{2}$ space of $\mathrm{Bun}_{\mathrm{GL}_{2}}$ of an elliptic curve. Alas, there are serious issues with this proposal (see Section 3.4).

1.7. There is, however, another possibility: rather than looking for the eigenfunctions of Hecke operators, one can look for the eigenfunctions of global differential operators on Bun . These eigenfunctions and the corresponding eigenvalues have been recently studied for $G=\mathrm{SL}_{2}$ by Joerg Teschner [50]. In a joint work with Pavel Etingof and David Kazhdan [16], we propose a canonical self-adjoint extension of the algebra of these differential operators and study the corresponding spectral problem. I discuss this proposal in Section 4.

According to a theorem of Beilinson and Drinfeld [6], there is a large commutative algebra of global holomorphic differential operators acting on sections of a square root $K^{1 / 2}$ of the canonical line bundle $K$ on $\operatorname{Bun}_{G}$ (this square root always exists, and is unique if $G$ is simplyconnected [6]). The complex conjugates of these differential operators are anti-holomorphic and act on sections of the complex conjugate line bundle $\bar{K}^{1 / 2}$ on $\operatorname{Bun}_{G}$. The tensor product of these two algebras is a commutative algebra acting on sections of the line bundle $K^{1 / 2} \otimes \bar{K}^{1 / 2}$ which we refer to as the bundle of half-densities on $\operatorname{Bun}_{G}$. 
The space of compactly supported sections of the line bundle $K^{1 / 2} \otimes \bar{K}^{1 / 2}$ on $\mathrm{Bun}_{G}$ (or rather, on its open dense subspace of stable $G$-bundles, provided that one exists) has a natural Hermitian inner product. Taking the completion of this space, we obtain a Hilbert space. Our differential operators are unbounded linear operators on this Hilbert space. We can ask whether these operators have natural self-adjoint extensions and if so, what are their joint eigenfunctions and eigenvalues. In Section 4.2, as a preview of [16], I give some more details on this construction. I then explain what happens in the abelian case of $G=\mathrm{GL}_{1}$ in Section 4.3.

In the case of $\mathrm{GL}_{1}$, the global differential operators are polynomials in the shift vector fields, holomorphic and anti-holomorphic, on the neutral component $\operatorname{Pic}^{0}(X)$ of the Picard variety of a complex curve $X$. These operators commute with each other (and with the Hecke operators, which are available in the abelian case), and their joint eigenfunctions are the standard Fourier harmonics on $\operatorname{Pic}^{0}(X)$. What about the eigenvalues? The spectrum of the commutative algebra of global holomorphic differential operators on $\operatorname{Pic}^{0}(X)$ can be identified with the space of holomorphic connections on the trivial line bundle on $X$. Hence every eigenvalue of this algebra can be encoded by a point in this space. It turns out that the points corresponding to the eigenvalues of this algebra on the space of $L^{2}$ functions on $\operatorname{Pic}^{0}(X)$ are precisely those holomorphic connections on the trivial line bundle on $X$ that give rise to the homomorphisms $\pi_{1}\left(X, p_{0}\right) \rightarrow \mathbb{C}^{\times}$ with image in $\mathbb{R}^{\times} \subset \mathbb{C}^{\times}$. In other words, these are the connections with monodromy in the split real form $\mathrm{GL}_{1}(\mathbb{R})$ of $\mathrm{GL}_{1}(\mathbb{C})$. This dovetails nicely with the conjecture of Teschner [50] in the case of $G=\mathrm{SL}_{2}$. We expect an analogous statement to hold for a general reductive group $G$, see [16].

Suppose for simplicity that $G$ is simply-connected. Then, according to a theorem of Beilinson and Drinfeld [6], the spectrum of the algebra of global holomorphic differential operators on $\operatorname{Bun}_{G}$ is canonically identified with the space of ${ }^{L} G$-opers on $X$. If $G=\mathrm{SL}_{2}$, then ${ }^{L} G=\mathrm{PGL}_{2}$ and $\mathrm{PGL}_{2}$-opers are the same as projective connections. Teschner [50] proposed that in this case, the eigenvalues correspond to the projective connections with monodromy taking values in the split real form $\mathrm{PGL}_{2}(\mathbb{R})$ of $\mathrm{PGL}_{2}(\mathbb{C})$ (up to conjugation by an element of $\mathrm{PGL}_{2}(\mathbb{C})$ ). Such projective connections have been described by W.M. Goldman [31]). For general $G$, we expect that the joint eigenvalues of the global holomorphic differential operators on $\operatorname{Bun}_{G}$ correspond to those ${ }^{L} G$-opers that have monodromy taking values in the split real form of ${ }^{L} G$ (up to conjugation). If so, then the spectra of the global differential operators on $\operatorname{Bun}_{G}$ can be described by analogues of the Langlands parameters of the classical theory: namely, certain homomorphisms from the fundamental group of $X$ to the Langlands dual group ${ }^{L} G$. A somewhat surprising element is that the homomorphisms that appear here are the ones whose image is in the split real form of ${ }^{L} G$ (rather than the compact form). More details will appear in $[16]$.

1.8. Thus, there is a rich analytic theory of joint eigenfunctions and eigenvalues of the global differential operators acting on half-densities on $\operatorname{Bun}_{G}$. This theory can be viewed as an analytic theory of automorphic functions for complex curves. So, Langlands was right to insist that an analytic theory exists, and he deserves a lot of credit for trying to construct it.

This raises the next question: what is the connection between this analytic theory and the geometric theory?

Valuable insights into this question may be gleaned from two-dimensional conformal field theory (CFT). In CFT, one has two types of correlation functions. The first type is chiral correlation functions, also known as conformal blocks. They form a vector space for fixed values of the parameters of the CFT. Hence we obtain a vector bundle of conformal blocks on the space of parameters. In addition, the data of conformal field theory give rise to a projectively flat connection on this bundle. The conformal blocks are multi-valued horizontal sections of this bundle. The second type is the "true" correlation functions. They can be expressed as sesquilinear combinations of conformal blocks and their complex conjugates (anti-conformal 
blocks), chosen so that the combination is a single-valued function of the parameters (see, e.g., $\left[29\right.$, Lecture 4]). ${ }^{3}$

Now, the Hecke eigensheaves on $\operatorname{Bun}_{G}$ constructed in [6] may be viewed as sheaves of conformal blocks of a certain two-dimensional conformal field theory, see [23]. Away from a singularity locus, these sheaves are vector bundles with a flat connection, and conformal blocks are their multi-valued horizontal sections (see Section 1.3 above). It turns out that in some cases there exist linear combinations of products of these conformal blocks and their complex conjugates which give rise to single-valued functions on $\operatorname{Bun}_{G}$. These functions are precisely the automorphic forms of the analytic theory. In other words, the objects of the analytic theory of automorphic forms on $\operatorname{Bun}_{G}$ can be constructed from the objects of the geometric Langlands theory in roughly the same way as the correlation functions of CFT are constructed from conformal blocks. This was predicted in [24] and [50]. An important difference with the CFT is that whereas in CFT the monodromy of conformal blocks is typically unitary, here we expect the monodromy to be in a split real group.

\section{The abelian case}

\subsection{The case of an elliptic curve}

Let's start with the case of an elliptic curve $E_{\tau}$ with complex parameter $\tau$. Let's choose, once and for all, a reference point $p_{0}$ on this curve. Then we can identify it with

$$
E_{\tau} \simeq \mathbb{C} /(\mathbb{Z}+\mathbb{Z} \tau)
$$

Next, consider the Picard variety $\operatorname{Pic}\left(E_{\tau}\right)$ of $E_{\tau}$. This is the (fine) moduli space of line bundles on $E_{\tau}$ (note that the corresponding moduli stack Bun $\mathrm{GL}_{1}\left(E_{\tau}\right)$ of line bundles on $E_{\tau}$ is the quotient of $\operatorname{Pic}\left(E_{\tau}\right)$ by the trivial action of the multiplicative group $\mathbb{G}_{m}=\mathrm{GL}_{1}$, which is the group of automorphisms of every line bundle on $E_{\tau}$ ). It is a disjoint union of connected components $\operatorname{Pic}^{d}\left(E_{\tau}\right)$ corresponding to line bundles of degree $d$. Using the reference point $p_{0}$, we can identity $\operatorname{Pic}^{d}\left(E_{\tau}\right)$ with $\operatorname{Pic}^{0}\left(E_{\tau}\right)$ by sending a line bundle $\mathcal{L}$ of degree $d$ to $\mathcal{L}\left(-d \cdot p_{0}\right)$. Furthermore, we can identify the degree 0 component $\operatorname{Pic}^{0}\left(E_{\tau}\right)$, which is the Jacobian variety of $E_{\tau}$, with $E_{\tau}$ itself using the Abel-Jacobi map; namely, we map a point $p \in E_{\tau}$ to the degree 0 line bundle $\mathcal{O}\left(p-p_{0}\right)$.

Now we define the Hecke operators $H_{p}$. They are labeled by points $p$ of the curve $E_{\tau}$. The operator $H_{p}$ is the pull-back of functions with respect to the geometric map

$$
\begin{aligned}
T_{p}: \operatorname{Pic}^{d}\left(E_{\tau}\right) & \rightarrow \operatorname{Pic}^{d+1}\left(E_{\tau}\right), \\
\mathcal{L} & \mapsto \mathcal{L}(p) .
\end{aligned}
$$

These operators commute with each other.

Formula (2.2) implies that if $f$ is a joint eigenfunction of the Hecke operators $H_{p}, p \in E_{\tau}$, on $\operatorname{Pic}\left(E_{\tau}\right)$, then its restriction $f_{0}$ to the connected component $\operatorname{Pic}^{0}\left(E_{\tau}\right)$ is an eigenfunction of the operators

$$
{ }_{p_{0}} H_{p}=H_{p_{0}}^{-1} H_{p},
$$

where $p_{0}$ is our reference point.

Conversely, given an eigenfunction $f_{0}$ of ${ }_{p_{0}} H_{p}, p \in X$, on $\operatorname{Pic}^{0}(X)$ and $\mu_{p_{0}} \in \mathbb{C}^{\times}$, there is a unique extension of $f_{0}$ to an eigenfunction $f$ of $H_{p}, p \in X$, such that the eigenvalue of $H_{p_{0}}$ on $f$

\footnotetext{
${ }^{3}$ As a useful analogy, consider the exponentials of harmonic functions, which may be written as products of holomorphic and anti-holomorphic functions.
} 
is equal to $\mu_{p_{0}}$. Namely, any line bundle $\mathcal{L}$ of degree $d$ may be represented uniquely as $\mathcal{L}_{0}\left(d \cdot p_{0}\right)$, where $\mathcal{L}_{0}$ is a line bundle of degree 0 . We then set

$$
f(\mathcal{L})=\left(\mu_{p_{0}}\right)^{d} \cdot f_{0}\left(\mathcal{L}_{0}\right)
$$

By construction, the eigenvalue $\mu_{p}$ of $H_{p}$ on $f$ is then equal to $\lambda_{p} \cdot \mu_{p_{0}}$, where $\lambda_{p}$ is the eigenvalue of ${ }_{p_{0}} H_{p}$ on $f_{0}$ (note that since $p_{0} H_{p_{0}}=\mathrm{Id}$, the eigenvalue $\lambda_{p_{0}}$ is always equal to 1 ).

Therefore, from now on we will consider the eigenproblem for the operators $p_{0} H_{p}$ acting on the space $L^{2}\left(\operatorname{Pic}^{0}\left(E_{\tau}\right)\right)$ of $L^{2}$-functions on $\operatorname{Pic}^{0}\left(E_{\tau}\right)$. Here, we define $L^{2}\left(\operatorname{Pic}^{0}\left(E_{\tau}\right)\right)$ as $L^{2}\left(E_{\tau}\right)$ (with respect to the measure on $E_{\tau}$ induced by the translation-invariant measure on $\mathbb{C}$ via the isomorphism (2.1)) using the above isomorphism between $\operatorname{Pic}^{0}\left(E_{\tau}\right)$ and $E_{\tau}$. The Hecke operator ${ }_{p_{0}} H_{p}$ acting on $L^{2}\left(E_{\tau}\right)$ is given by the formula

$$
\left({ }_{p_{0}} H_{p} \cdot f\right)(q)=f(q+p)
$$

In other words, it is simply the pull-back under the shift by $p$ with respect to the (additive) abelian group structure on $E_{\tau}$, which can be described explicitly using the isomorphism (2.1). The subscript $p_{0}$ in $p_{0} H_{p}$ serves as a reminder that this operator depends on the choice of the reference point $p_{0}$.

Now we would like to describe the joint eigenfunctions and eigenvalues of the operators ${ }_{p_{0}} H_{p}$ on $L^{2}\left(E_{\tau}\right)$.

To be even more concrete, let's start with the case $\tau=\mathrm{i}$, so $E_{\tau}=E_{\mathrm{i}}$ which is identified with $\mathbb{C} /(\mathbb{Z}+\mathbb{Z i})$ as above. Thus, we have a measure-preserving isomorphism between $E_{\mathrm{i}}$ and the product of two circles $(\mathbb{R} / \mathbb{Z}) \times(\mathbb{R} / \mathbb{Z})$ corresponding to the real and imaginary parts of $z=x+\mathrm{i} y$. The space of $L^{2}$ functions on the curve $E_{\mathrm{i}}$ is therefore the completed tensor product of two copies of $L^{2}(\mathbb{R} / \mathbb{Z})$, and so it has the standard orthogonal Fourier basis:

$$
f_{m, n}(x, y)=\mathrm{e}^{2 \pi \mathrm{i} m x} \cdot \mathrm{e}^{2 \pi \mathrm{i} n y}, \quad m, n \in \mathbb{Z} .
$$

Let us write $p=x_{p}+y_{p} \mathrm{i} \in E_{\mathrm{i}}$, with $x_{p}, y_{p} \in[0,1)$. The operator ${ }_{p_{0}} H_{p}$ corresponds to the shift of $z$ by $p$ (with respect to the abelian group structure on $E_{\mathrm{i}}$ ):

$$
\left({ }_{p_{0}} H_{p} \cdot f\right)(x, y)=f\left(x+x_{p}, y+y_{p}\right), \quad f \in L^{2}\left(E_{\mathrm{i}}\right) .
$$

It might be instructive to consider first the one-dimensional analogue of this picture, in which we have $L^{2}\left(S^{1}\right)$, where $S_{1}=\mathbb{C} / \mathbb{Z}$ with coordinate $\phi$. Then the role of the family $\left\{{ }_{p_{0}} H_{p}\right\}_{p \in E_{\mathrm{i}}}$ is played by the family $\left\{H_{\alpha}^{\prime}\right\}_{\alpha \in S^{1}}$ acting by shifts:

$$
\left(H_{\alpha}^{\prime} \cdot f\right)(x)=f(\phi+\alpha), \quad f \in L^{2}\left(S^{1}\right) .
$$

Then the Fourier harmonics $f_{n}(x)=\mathrm{e}^{2 \pi \mathrm{in} \phi}$ form an orthogonal eigenbasis of the operators $H_{\alpha}^{\prime}$, $\alpha \in S^{1}$. The eigenvalue of $H_{\alpha}^{\prime}$ on $f_{n}$ is $\mathrm{e}^{2 \pi \mathrm{in} \alpha}$.

Likewise, in the two-dimensional case of the elliptic curve $E_{\mathrm{i}}$, the Fourier harmonics $f_{m, n}$ form an orthogonal basis of eigenfunctions of the operators ${ }_{p_{0}} H_{p}, p \in E_{\mathrm{i}}$, in $L^{2}\left(E_{\mathrm{i}}\right)$ :

$$
{ }_{p_{0}} H_{p} \cdot f_{m, n}=\mathrm{e}^{2 \pi \mathrm{i}\left(m x_{p}+n y_{p}\right)} f_{m, n} .
$$

From this formula we see that the eigenvalue of ${ }_{p_{0}} H_{p}$ on $f_{m, n}$ is $\mathrm{e}^{2 \pi \mathrm{i}\left(m x_{p}+n y_{p}\right)}$. Thus, we have obtained a complete description of the Hecke eigenfunctions and eigenvalues for the curve $X=E_{\mathrm{i}}$ and the group $G=\mathrm{GL}_{1}$. 


\subsection{General elliptic curve}

Now we generalize this to the case of an arbitrary elliptic curve $E_{\tau} \simeq \mathbb{C} /(\mathbb{Z}+\mathbb{Z} \tau)$ with $\operatorname{Im} \tau>0$. Recall that we identify every component of $\operatorname{Pic}\left(E_{\tau}\right)$ with $E_{\tau}$ using the reference point $p_{0}$. Then we obtain the Hecke operators ${ }_{p_{0}} H_{p}$ labeled by $p \in E_{\tau}$ given by the shift by $p$ naturally acting on $E_{\tau}$ (see formula (2.3)). The eigenfunctions and eigenvalues of these operators are then given by the following theorem.

Theorem 2.1. The joint eigenfunctions of the Hecke operators ${ }_{p_{0}} H_{p}, p \in E_{\tau}$, on $L^{2}\left(E_{\tau}\right)$ are

$$
f_{m, n}^{\tau}(z, \bar{z})=\mathrm{e}^{2 \pi \mathrm{i} m(z \bar{\tau}-\bar{z} \tau) /(\bar{\tau}-\tau)} \cdot \mathrm{e}^{2 \pi \mathrm{i} n(z-\bar{z}) /(\tau-\bar{\tau})}, \quad m, n \in \mathbb{Z} .
$$

The eigenvalues are given by the right hand side of the following formula:

$$
{ }_{p_{0}} H_{p} \cdot f_{m, n}^{\tau}=\mathrm{e}^{2 \pi \mathrm{i} m(p \bar{\tau}-\bar{p} \tau) /(\bar{\tau}-\tau)} \cdot \mathrm{e}^{2 \pi \mathrm{i} n(p-\bar{p}) /(\tau-\bar{\tau})} f_{m, n}^{\tau} .
$$

In Section 2.4 we will give an alternative formula for these eigenfunctions (for an arbitrary smooth projective curve instead of $E_{\tau}$ ).

\subsection{Digression: Eigenvalues of the Hecke operators and representations of the fundamental group}

Let $\mathbf{H}\left(E_{\tau}\right)$ be the spectrum of the algebra of Hecke operators acting on $L^{2}\left(\operatorname{Pic}^{0}\left(E_{\tau}\right)\right)=L^{2}\left(E_{\tau}\right)$. In this subsection we compare the description of $\mathbf{H}\left(E_{\tau}\right)$ given in Theorem 2.1 with that envisioned by Langlands in [42].

Let $\mathbf{E}\left(E_{\tau}\right)$ be the set of equivalence classes of one-dimensional representations of the fundamental group $\pi_{1}\left(E_{\tau}, p_{0}\right)$ with finite image. In [42], Langlands attempts to construct a one-to-one correspondence between $\mathbf{H}\left(E_{\tau}\right)$ and $\mathbf{E}\left(E_{\tau}\right)$ in two different ways.

The first is to express the Hecke eigenvalues corresponding to a given Hecke eigenfunction as holonomies of a flat unitary connection on a line bundle on $E_{\tau}$ and then take the monodromy representation of this connection (see part (4) in Section 1.4). I show below that it is indeed possible to express the Hecke eigenvalues that we have found in Theorem 2.1 as holonomies of a flat unitary connection on the trivial line bundle on $E_{\tau}$ (furthermore, this will be generalized in Section 2.4 to the case of an arbitrary curve $X)$. But all of these connections have trivial monodromy representation. Thus, the map $\mathbf{H}\left(E_{\tau}\right) \rightarrow \mathbf{E}\left(E_{\tau}\right)$ we obtain this way is trivial, i.e., its image consists of a single element of $\mathbf{E}\left(E_{\tau}\right)$. (Herein lies an important difference between the analytic and geometric theories for curves over $\mathbb{C}$, which is discussed in more detail in Remark 2.3 below.)

Second, Langlands attempted to construct a map $\mathbf{H}\left(E_{\tau}\right) \rightarrow \mathbf{E}\left(E_{\tau}\right)$ explicitly. Unfortunately, this construction does not yield a bijective map, either, as I show in Remark 2.2 below.

Let me show how to express the eigenvalues of the Hecke operators $p_{0} H_{p}, p \in E_{\tau}$, on a given eigenfunction as holonomies of a flat unitary connection.

Consider first the case of $\tau=\mathrm{i}$. In this case, we assign to the Hecke eigenfunction $f_{m, n}$ given by formula (2.4) the following unitary flat connection $\nabla^{(m, n)}$ on the trivial line bundle over $E_{\mathrm{i}}$ :

$$
\nabla^{(m, n)}=d-2 \pi \mathrm{i} m \mathrm{~d} x-2 \pi \mathrm{i} n \mathrm{~d} y
$$

(since the line bundle is trivial, a connection on it is the same as a one-form on the curve). In other words, the corresponding first order differential operators along $x$ and $y$ are given by the formulas

$$
\nabla_{x}^{(m, n)}=\frac{\partial}{\partial x}-2 \pi \mathrm{i} m, \quad \nabla_{y}^{(m, n)}=\frac{\partial}{\partial y}-2 \pi \mathrm{i} n .
$$


The horizontal sections of this connection are the solutions of the equations

$$
\nabla_{x}^{(m, n)} \cdot \Phi=\nabla_{y}^{(m, n)} \cdot \Phi=0
$$

They have the form

$$
\Phi_{m, n}(x, y)=\mathrm{e}^{2 \pi \mathrm{i}(m x+n y)}
$$

up to a scalar. The function $\Phi_{m, n}$ is the unique solution of (2.6) normalized so that its value at the point $0 \in E_{\mathrm{i}}$, corresponding to our reference point $p_{0} \in E_{\mathrm{i}}$, is equal to 1 . The value of this function $\Phi_{m, n}$ at $p=x_{p}+\mathrm{i} y_{p} \in \mathbb{C} /(\mathbb{Z}+\mathbb{Z i})$ is indeed equal to the eigenvalue of the Hecke operator ${ }_{p_{0}} H_{p}$ on the harmonic $f_{m, n}$.

Thus, this eigenvalue can be represented as the holonomy of the connection $\nabla^{(m, n)}$ over a path connecting our reference point $p_{0} \in E_{\mathrm{i}}$, which corresponds to $0 \in \mathbb{C} /(\mathbb{Z}+\mathbb{Z i})$, and the point $p \in E_{\mathrm{i}}$. Since the connection is flat, it does not matter which path we choose.

However, and this is a crucial point, the connection $\nabla^{(m, n)}$ has trivial monodromy on $E_{\mathrm{i}}$. Indeed,

$$
\Phi_{m, n}(x+1, y)=\Phi_{m, n}(x, y+1)=\Phi_{m, n}(x, y)
$$

for all $m, n \in \mathbb{Z}$.

Similarly, we assign a flat unitary connection ${ }_{\tau} \nabla^{(m, n)}$ on the trivial line bundle on $E_{\tau}$ for each Hecke eigenfunction $f_{m, n}^{\tau}$ :

$$
{ }_{\tau} \nabla^{(m, n)}=d-2 \pi \mathrm{i} \frac{n-m \bar{\tau}}{\tau-\bar{\tau}} \mathrm{d} z-2 \pi \mathrm{i} \frac{m \tau-n}{\tau-\bar{\tau}} \mathrm{d} \bar{z} .
$$

The first order operators corresponding to $z$ and $\bar{z}$ are

$$
\begin{aligned}
{ }_{\tau} \nabla_{z}^{(m, n)} & =\frac{\partial}{\partial z}-2 \pi \mathrm{i} \frac{n-m \bar{\tau}}{\tau-\bar{\tau}}, \\
{ }_{\tau} \nabla_{\bar{z}}^{(m, n)} & =\frac{\partial}{\partial \bar{z}}-2 \pi \mathrm{i} \frac{m \tau-n}{\tau-\bar{\tau}} .
\end{aligned}
$$

Just as in the case $\tau=\mathrm{i}$, for every $p \in E_{\tau}$, the holonomy of the connection ${ }_{\tau} \nabla^{(m, n)}$ over a path connecting $p_{0} \in E_{\tau}$ and $p \in E_{\tau}$ is equal to the eigenvalue of ${ }_{p_{0}} H_{p}$ on $f_{m, n}^{\tau}$ given by the right hand side of formula (2.5). However, as in the case of $\tau=\mathrm{i}$, all connections ${ }_{\tau} \nabla^{(m, n)}$ yield the trivial monodromy representation $\pi_{1}\left(E_{\tau}, p_{0}\right) \rightarrow \mathrm{GL}_{1}$.

Remark 2.2. On pp. 59-60 of [42], another attempt is made to construct a map from the set $\mathbf{H}\left(E_{\tau}\right)$ (the spectrum of the algebra of Hecke operators acting on $L^{2}\left(E_{\tau}\right)$ ) to the set $\mathbf{E}\left(E_{\tau}\right)$ of equivalence classes of homomorphisms $\pi_{1}\left(E_{\tau}, p_{0}\right) \rightarrow \mathrm{GL}_{1}$ with finite image. According to Theorem 2.1, the set $\mathbf{H}\left(E_{\tau}\right)$ is identified with $\mathbb{Z} \times \mathbb{Z}$. On the other hand, the set $\mathbf{E}\left(E_{\tau}\right)$ can be identified with $\mu \times \mu$, where $\mu$ is the group of complex roots of unity (we have an isomorphism $\mathbb{Q} / \mathbb{Z} \simeq \mu$ sending $\kappa \in \mathbb{Q} / \mathbb{Z}$ to $\left.\mathrm{e}^{2 \pi \mathrm{i} \kappa}\right)$. Indeed, since $\pi_{1}\left(E_{\tau}, p_{0}\right) \simeq \mathbb{Z} \times \mathbb{Z}$, a homomorphism $\phi: \pi_{1}\left(E_{\tau}, p_{0}\right) \rightarrow \mathrm{GL}_{1} \simeq \mathbb{C}^{\times}$is uniquely determined by its values on the elements $A=(1,0)$ and $B=(0,1)$ of $\mathbb{Z} \times \mathbb{Z}$. The homomorphism $\phi$ has finite image if and only if both $\phi(A), \phi(B)$ belong to $\mu$.

Langlands attempts to construct a map $(\mathbb{Z} \times \mathbb{Z}) \rightarrow(\mu \times \mu)$ as follows (see pp. 59-60 of [42]): he sets

$$
(0,0) \mapsto(1,1)
$$


Next, given a non-zero element $(k, l) \in \mathbb{Z} \times \mathbb{Z}$, there exists a matrix $g_{k, l}=\left(\begin{array}{ll}\alpha & \beta \\ \gamma & \delta\end{array}\right) \in \mathrm{SL}_{2}(\mathbb{Z})$ such that

$$
\left(\begin{array}{ll}
k & l
\end{array}\right)=\left(\begin{array}{ll}
k^{\prime} & 0
\end{array}\right)\left(\begin{array}{ll}
\alpha & \beta \\
\gamma & \delta
\end{array}\right), \quad k^{\prime}>0
$$

Two comments on (2.7): first, as noted in [42], the matrix $g_{k, l}$ is not uniquely determined by formula (2.7). Indeed, this formula will still be satisfied if we multiply $g_{k, l}$ on the left by any lower triangular matrix in $\mathrm{SL}_{2}(\mathbb{Z})$. Second, formula (2.7) implies that

$$
(k, l)=k^{\prime}(\alpha, \beta), \quad \operatorname{gcd}(\alpha, \beta)= \pm 1, \quad k^{\prime}>0,
$$

where, for a pair of integers $(k, l) \neq(0,0)$, we define $\operatorname{gcd}(k, l)$ as $l$ if $k=0$, as $k$ if $l=0$, and $\operatorname{gcd}(|k|,|l|)$ times the product of the signs of $k$ and $l$ if they are both non-zero. Therefore

$$
k^{\prime}=|\operatorname{gcd}(k, l)| .
$$

Using a particular choice of the matrix $g_{k, l}$, Langlands defines a new set of generators $\left\{A^{\prime}, B^{\prime}\right\}$ of the group $\pi_{1}\left(E_{\tau}, p_{0}\right)$ :

$$
A^{\prime}=A^{\alpha} B^{\beta}, \quad B^{\prime}=A^{\gamma} B^{\delta} .
$$

He then defines a homomorphism $\phi_{k, l}: \pi_{1}\left(E_{\tau}, p_{0}\right) \rightarrow \mathrm{GL}_{1}$ corresponding to $(k, l)$ by the formulas

$$
A^{\prime} \mapsto \mathrm{e}^{2 \pi \mathrm{i} / k^{\prime}}, \quad B^{\prime} \mapsto 1
$$

Now, formula (2.9) implies that

$$
A=\left(A^{\prime}\right)^{\delta}\left(B^{\prime}\right)^{-\beta}, \quad B=\left(A^{\prime}\right)^{-\gamma}\left(B^{\prime}\right)^{\alpha},
$$

and so we find the values of $\phi_{k, l}$ on the original generators $A$ and $B$ :

$$
A \mapsto \mathrm{e}^{2 \pi \mathrm{i} \delta / k^{\prime}}, \quad B \mapsto \mathrm{e}^{-2 \pi \mathrm{i} \gamma / k^{\prime}} .
$$

Langlands writes in [42], "This has a peculiar property that part of the numerator becomes the denominator, which baffles me and may well baffle the reader". He goes on to say, "To be honest, this worries me".

In fact, this construction does not give us a well-defined map $(\mathbb{Z} \times \mathbb{Z}) \rightarrow(\mu \times \mu)$. Indeed, $g_{k, l}$ is only defined up to left multiplication by a lower triangular matrix:

$$
\left(\begin{array}{ll}
\alpha & \beta \\
\gamma & \delta
\end{array}\right) \mapsto\left(\begin{array}{ll}
1 & 0 \\
x & 1
\end{array}\right)\left(\begin{array}{ll}
\alpha & \beta \\
\gamma & \delta
\end{array}\right), \quad x \in \mathbb{Z}
$$

under which we have the following transformation:

$$
\gamma \mapsto \gamma+x \alpha, \quad \delta \mapsto \delta+x \beta
$$

But then the homomorphism (2.10) gets transformed to the homomorphism sending

$$
A \mapsto \mathrm{e}^{2 \pi \mathrm{i}(\delta+x \beta) / k^{\prime}}, \quad B \mapsto \mathrm{e}^{-2 \pi \mathrm{i}(\gamma+x \alpha) / k^{\prime}} .
$$

The homomorphisms (2.10) and (2.11) can only coincide for all $x \in \mathbb{Z}$ if both $\alpha$ and $\beta$ are divisible by $k^{\prime}$. But if $k^{\prime} \neq 1$, this contradicts the condition, established in formula (2.8), that $\alpha$ and $\beta$ are relatively prime. Hence (2.10) and (2.11) will in general differ from each other, and so we don't get a well-defined map $(\mathbb{Z} \times \mathbb{Z}) \rightarrow(\mu \times \mu)$. 
We could try to fix this problem by replacing the relation (2.9) with

$$
A=\left(A^{\prime}\right)^{\alpha}\left(B^{\prime}\right)^{\gamma}, \quad B=\left(A^{\prime}\right)^{\beta}\left(B^{\prime}\right)^{\delta} .
$$

Then the homomorphism $\phi_{k, l}$ would send

$$
A \mapsto \mathrm{e}^{2 \pi \mathrm{i} \alpha / k^{\prime}}, \quad B \mapsto \mathrm{e}^{2 \pi \mathrm{i} \beta / k^{\prime}} .
$$

This way, we get a well-defined map $(\mathbb{Z} \times \mathbb{Z}) \rightarrow(\mu \times \mu)$, but it's not a bijection.

In fact, there is no reason to expect that there is a meaningful bijection between the above sets $\mathbf{H}\left(E_{\tau}\right)$ and $\mathbf{E}\left(E_{\tau}\right)$. Indeed, according to Theorem 2.1, the set $\mathbf{H}\left(E_{\tau}\right)$ can be naturally identified with the group of continuous characters $E_{\tau} \rightarrow \mathbb{C}^{\times}$(where $E_{\tau}$ is viewed as an abelian group), which is isomorphic to $\mathbb{Z} \times \mathbb{Z}$.

On the other hand, let $\mathbf{E}\left(E_{\tau}\right)$ is the subgroup of elements of finite order in the group of characters $\pi_{1}\left(E_{\tau}, p_{0}\right) \rightarrow \mathbb{C}^{\times}$. The whole group of such characters, which is isomorphic to $\mathbb{C}^{\times} \times \mathbb{C}^{\times}$, is the dual group of $\mathbb{Z} \times \mathbb{Z}=\mathbf{H}\left(E_{\tau}\right)$. The set $\mathbf{E}\left(E_{\tau}\right)$ is its subgroup of elements of finite order, which isomorphic to $\mu \times \mu$, where $\mu$ is the (multiplicative) group of complex roots of unity. Clearly, $\mathbb{Z} \times \mathbb{Z}$ and $\mu \times \mu$ are not isomorphic as abstract groups. Of course, since each of these two sets is countable, there exist bijections between them as sets. But it's hard to imagine that such a bijection would be pertinent to the questions at hand.

Remark 2.3. Recall that in the classical unramified Langlands correspondence for a curve over $\mathbb{F}_{q}$, to each joint eigenfunction of the Hecke operators we assign a Langlands parameter. In the case of $G=\mathrm{GL}_{n}$, this is an equivalence class of $\ell$-adic homomorphisms from the étale fundamental group of $X$ to $\mathrm{GL}_{n}$ (and more generally, one considers homomorphisms to the Langlands dual group ${ }^{L} G$ of $G$ ). Given such a homomorphism $\sigma$, to each closed point $x$ of $X$ we can assign an $\ell$-adic number, the trace of $\sigma\left(\mathrm{Fr}_{x}\right)$, where $\operatorname{Fr}_{x}$ is the Frobenius conjugacy class, so we obtain a function from the set of closed points of $X$ to the set of conjugacy classes in $\mathrm{GL}_{n}\left(\overline{\mathbb{Q}}_{\ell}\right)$.

In the geometric Langlands correspondence for curves over $\mathbb{C}$, the picture is different. Now the role of the étale fundamental group is played by the topological fundamental group $\pi_{1}\left(X, p_{0}\right)$. Thus, the Langlands parameters are the equivalence classes of homomorphisms $\pi_{1}\left(X, p_{0}\right) \rightarrow \mathrm{GL}_{n}$ (or, more generally, to ${ }^{L} G$ ). The question then is: how to interpret such a homomorphism as a Hecke "eigenvalue" on a Hecke eigensheaf?

The point is that for a Hecke eigensheaf, the "eigenvalue" of a Hecke operator (or rather, Hecke functor) is not a number but an $n$-dimensional vector space. As we move along a closed path on our curve (starting and ending at the point $p_{0}$ say), this vector space will in general undergo a non-trivial linear transformation, thus giving rise to a non-trivial homomorphism $\pi_{1}\left(X, p_{0}\right) \rightarrow \mathrm{GL}_{n}$

Note that over $\mathbb{C}$ we have the Riemann-Hilbert correspondence, which sets up a bijection between the set of equivalence classes of homomorphisms $\pi_{1}\left(X, p_{0}\right) \rightarrow \mathrm{GL}_{n}$ (or, more generally, $\left.\pi_{1}\left(X, p_{0}\right) \rightarrow{ }^{L} G\right)$ and the set of equivalence classes of pairs $(\mathcal{P}, \nabla)$, where $\mathcal{P}$ is a rank $n$ bundle on $X$ (or, more generally, an ${ }^{L} G$-bundle) and $\nabla$ is a flat connection on $\mathcal{P}$. The map between the two data is defined by assigning to $(\mathcal{P}, \nabla)$ the monodromy representation of $\nabla$ (corresponding to a specific a trivialization of $\mathcal{P}$ at $\left.p_{0}\right)$. We may therefore take equivalence classes of the flat bundles $(\mathcal{P}, \nabla)$ as our Langlands parameters instead of equivalence classes of homomorphisms $\pi_{1}\left(X, p_{0}\right) \rightarrow \mathrm{GL}_{n}$. As explained in the previous paragraph, these flat bundles $(\mathcal{P}, \nabla)$ will in general have non-trivial monodromy.

However, in this section we consider (in the case of $\mathrm{GL}_{1}$ and a curve $X$ ) the eigenfunctions of the Hecke operators ${ }_{p_{0}} H_{p}, p \in X$, on $\operatorname{Pic}^{0}(X)$. Their eigenvalues are numbers, not vector spaces. Therefore they cannot undergo any transformations as we move along a closed path on 
our curve. In other words, these numbers give rise to a single-valued function from $X$ to $\mathrm{GL}_{1}(\mathbb{C})$ (it actually takes values in $U_{1} \subset \mathrm{GL}_{1}(\mathbb{C})$ ). Because the function is single-valued, if we represent this function as the holonomy of a flat connection on a line bundle on $X$, then this connection necessarily has trivial monodromy. And indeed, we have seen above that each collection of joint eigenvalues of the Hecke operators ${ }_{p_{0}} H_{p}, p \in E_{\tau}$, on functions on $\operatorname{Pic}^{0}\left(E_{\tau}\right)$ can be represented as holonomies of a specific (unitary) connection ${ }_{\tau} \nabla^{m, n}$ with trivial monodromy. The same is true for other curves, as we will see below.

\subsection{Higher genus curves}

Let $X$ be a smooth projective connected curve over $\mathbb{C}$. Denote by $\operatorname{Pic}(X)$ the Picard variety of $X$, i.e., the moduli space of line bundles on $X$ (as before, the moduli stack $\operatorname{Bun}_{\mathrm{GL}_{1}}(X)$ of line bundles on $X$ is the quotient of $\operatorname{Pic}(X)$ by the trivial action of $\left.\mathbb{G}_{m}=\mathrm{GL}_{1}\right)$. We have a decomposition of $\operatorname{Pic}(X)$ into a disjoint union of connected components $\operatorname{Pic}^{d}(X)$ corresponding to line bundles of degree $d$. The Hecke operator $H_{p}, p \in X$, is the pull-back of functions with respect to the map (see formula $(2.2)$ for $X=E_{\tau}$ ):

$$
\begin{aligned}
T_{p}: \operatorname{Pic}^{d}(X) & \rightarrow \operatorname{Pic}^{d+1}(X), \\
\mathcal{L} & \mapsto \mathcal{L}(p) .
\end{aligned}
$$

The Hecke operators $H_{p}$ with different $p \in X$ commute with each other, and it is natural to consider the problem of finding joint eigenfunctions and eigenvalues of these operators on functions on $\operatorname{Pic}(X)$. In the same way as in Section 2.1, we find that this problem is equivalent to the problem of finding joint eigenfunctions and eigenvalues of the operators ${ }_{p_{0}} H_{p}=H_{p_{0}}^{-1} H_{p}$ on functions on $\operatorname{Pic}^{0}(X)$, where $p_{0}$ is a reference point on $X$ that we choose once and for all. The operator ${ }_{p_{0}} H_{p}$ is the pull-back of functions with respect to the map $p_{0} T_{p}: \operatorname{Pic}^{0}(X) \rightarrow \operatorname{Pic}^{0}(X)$ sending a line bundle $\mathcal{L}$ to $\mathcal{L}\left(p-p_{0}\right)$.

Now, $\operatorname{Pic}^{0}(X)$ is the Jacobian of $X$, which is a $2 g$-dimensional torus (see, e.g., [33])

$$
\operatorname{Pic}^{0}(X) \simeq H^{0}\left(X, \Omega^{1,0}\right)^{*} / H_{1}(X, \mathbb{Z}),
$$

where $H_{1}(X, \mathbb{Z})$ is embedded into the space of linear functionals on the space $H^{0}\left(X, \Omega^{1,0}\right)$ of holomorphic one-forms on $X$ by sending $\beta \in H_{1}(X, \mathbb{Z})$ to the linear functional

$$
\omega \in H^{0}\left(X, \Omega^{1,0}\right) \mapsto \int_{\beta} \omega .
$$

Motivated by Theorem 2.1, it is natural to guess that the standard Fourier harmonics in $L^{2}\left(\operatorname{Pic}^{0}(X)\right)$ form an orthogonal eigenbasis of the Hecke operators. This is indeed the case.

To see that, we give an explicit formula for these harmonics. They can be written in the form $\mathrm{e}^{2 \pi \mathrm{i} \varphi}$, where $\varphi: H^{0}\left(X, \Omega^{1,0}\right)^{*} \rightarrow \mathbb{R}$ is an $\mathbb{R}$-linear functional such that $\varphi(\beta) \in \mathbb{Z}$ for all $\beta \in H_{1}(X, \mathbb{Z})$. To write them down explicitly, we use the Hodge decomposition

$$
H^{1}(X, \mathbb{C})=H^{0}\left(X, \Omega^{1,0}\right) \oplus H^{0}\left(X, \Omega^{0,1}\right)=H^{0}\left(X, \Omega^{1,0}\right) \oplus \overline{H^{0}\left(X, \Omega^{1,0}\right)}
$$

to identify $H^{0}\left(X, \Omega^{1,0}\right)$, viewed as an $\mathbb{R}$-vector space, with $H^{1}(X, \mathbb{R})$ by the formula

$$
\omega \in H^{0}\left(X, \Omega^{1,0}\right) \mapsto \omega+\bar{\omega} .
$$

In particular, for any class $c \in H^{1}(X, \mathbb{R})$, there is a unique holomorphic one-form $\omega_{c}$ such that $c$ is represented by the real-valued harmonic one-form $\omega_{c}+\bar{\omega}_{c}$,

$$
H^{1}(X, \mathbb{R}) \ni c=\omega_{c}+\bar{\omega}_{c}, \quad \omega_{c} \in H^{0}\left(X, \Omega^{1,0}\right) .
$$


Viewed as a real manifold,

$$
\operatorname{Pic}^{0}(X) \simeq H^{1}(X, \mathbb{R})^{*} / H_{1}(X, \mathbb{Z}),
$$

where $H_{1}(X, \mathbb{Z})$ is embedded into $H^{1}(X, \mathbb{R})^{*}$ by sending $\beta \in H_{1}(X, \mathbb{Z})$ to the linear functional on $H^{1}(X, \mathbb{R})$ given by the formula (compare with formulas (2.12) and (2.13))

$$
H^{1}(X, \mathbb{R}) \ni c \mapsto \int_{\beta} c=\int_{\beta}\left(\omega_{c}+\bar{\omega}_{c}\right) .
$$

Now, to each $\gamma \in H^{1}(X, \mathbb{Z})$ we attach the corresponding element of the vector space $H^{1}(X, \mathbb{R})$, which can be viewed as a linear functional $\varphi_{\gamma}$ on the dual vector space $H^{1}(X, \mathbb{R})^{*}$,

$$
\varphi_{\gamma}: H^{1}(X, \mathbb{R})^{*} \rightarrow \mathbb{R}
$$

It has the desired property: $\varphi_{\gamma}(\beta) \in \mathbb{Z}$ for all $\beta \in H_{1}(X, \mathbb{Z})$. The corresponding functions

$$
\mathrm{e}^{2 \pi \mathrm{i} \varphi_{\gamma}}, \quad \gamma \in H^{1}(X, \mathbb{Z}),
$$

are the Fourier harmonics that form an orthogonal basis of the Hilbert space $L^{2}\left(\operatorname{Pic}^{0}(X)\right)$.

We claim that each of these functions is an eigenfunction of the Hecke operators $p_{0} H_{p}, p \in X$, so that together they give us a sought-after orthogonal eigenbasis of the Hecke operators. To see that, we use the Abel-Jacobi map.

For $d>0$, let $X^{(d)}$ be the $d$ th symmetric power of $X$, and $p_{d}: X^{(d)} \rightarrow \operatorname{Pic}^{d}(X)$ the AbelJacobi map

$$
p_{d}(D)=\mathcal{O}(D), \quad D=\sum_{i=1}^{d}\left[x_{i}\right], \quad x_{i} \in X
$$

We can lift the map $T_{p}$ to a map

$$
\begin{aligned}
\widetilde{T}_{p}: X^{(d)} & \rightarrow X^{(d+1)}, \\
D & \mapsto D+[p],
\end{aligned}
$$

so that we have a commutative diagram

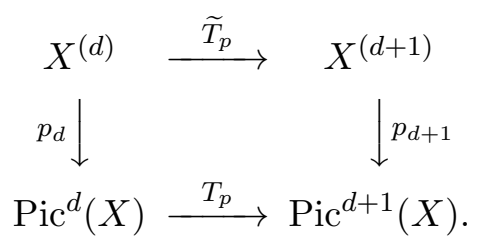

Denote by $\widetilde{H}_{p}$ the corresponding pull-back operator on functions.

Now let $f_{0}$ be a non-zero function on $\operatorname{Pic}^{0}(X)$. Identifying $\operatorname{Pic}^{d}(X)$ with $\operatorname{Pic}^{0}(X)$ using the reference point $p_{0}$ :

$$
\mathcal{L} \mapsto \mathcal{L}\left(-d \cdot p_{0}\right)
$$

we obtain a non-zero function $f_{d}$ on $\operatorname{Pic}^{d}(X)$ for all $d \in \mathbb{Z}$. Let $\widetilde{f}_{d}$ the pull-back of $f_{d}$ to $X^{(d)}$ for $d>0$. Suppose that these functions satisfy

$$
\widetilde{H}_{p}\left(\widetilde{f}_{d+1}\right)=\lambda_{p} \widetilde{f}_{d}, \quad p \in X, \quad d>0,
$$


where $\lambda_{p} \neq 0$ for all $p$ and $\lambda_{p_{0}}=1$. This is equivalent to the following factorization formula for $\widetilde{f}_{d}$ :

$$
\widetilde{f}_{d}\left(\sum_{i=1}^{d}\left[x_{i}\right]\right)=c \prod_{i=1}^{d} \lambda_{x_{i}}, \quad c \in \mathbb{C}, \quad d>0 .
$$

The surjectivity of $p_{d}$ with $d \geq g$ and the commutativity of the diagram (2.16) then implies that

$$
H_{p}\left(f_{d+1}\right)=\lambda_{p} f_{d}, \quad p \in X, \quad d \geq g .
$$

But then it follows from the definition of $f_{d}$ that $f_{0}$ is an eigenfunction of the operators ${ }_{p_{0}} H_{p}=$ $H_{p_{0}}^{-1} H_{p}$ with the eigenvalues $\lambda_{p}=\widetilde{f}_{1}([p])$.

This observation gives us an effective way to demonstrate that a given function $f_{0}$ on $\operatorname{Pic}^{0}(X)$ is a Hecke eigenfunction.

Let us use it in the case of the function $f_{0}=\mathrm{e}^{2 \pi i \varphi_{\gamma}}, \gamma \in H^{1}(X, \mathbb{Z})$, on $\operatorname{Pic}^{0}(X)$ given by formula (2.15). For that, denote by

$$
{ }_{d} \mathrm{e}^{2 \pi \mathrm{i} \varphi_{\gamma}}, \quad \gamma \in H^{1}(X, \mathbb{Z}),
$$

the corresponding functions $f_{d}$ on $\operatorname{Pic}^{d}(X)$ obtained via the identification (2.17). We claim that for any $\gamma \in H^{1}(X, \mathbb{Z})$, the pull-backs of ${ }_{d} \mathrm{e}^{2 \pi \mathrm{i} \varphi_{\gamma}}$ to $X^{(d)}, d>0$, via the Abel-Jacobi maps have the form (2.18), and hence $\mathrm{e}^{2 \pi \mathrm{i} \varphi_{\gamma}}$ is a Hecke eigenfunction on $\operatorname{Pic}^{0}(X)$.

To see that, we recall an explicit formula for the composition

$$
X^{(d)} \rightarrow \operatorname{Pic}^{d}(X) \rightarrow \operatorname{Pic}^{0}(X) \simeq H^{0}\left(X, \Omega^{1,0}\right)^{*} / H_{1}(X, \mathbb{Z}),
$$

where the second map is given by formula (2.17) (see, e.g., [33]). Namely, the composition (2.20) maps $\sum_{i=1}^{d}\left[x_{i}\right] \in X^{(d)}$ to the linear functional on $H^{0}\left(X, \Omega^{1,0}\right)$ sending

$$
\omega \in H^{0}\left(X, \Omega^{1,0}\right) \mapsto \sum_{i=1}^{d} \int_{p_{0}}^{x_{i}} \omega .
$$

Composing the map (2.20) with the isomorphism $H^{0}\left(X, \Omega^{1,0}\right) \simeq H^{1}(X, \mathbb{R})$ defined above, we obtain a map

$$
{ }_{p_{0}} \Phi_{d}: X^{(d)} \rightarrow H^{1}(X, \mathbb{R})^{*} / H_{1}(X, \mathbb{Z}),
$$

which maps $\sum_{i=1}^{d}\left[x_{i}\right] \in X^{(d)}$ to the linear functional ${ }_{p_{0}} \Phi_{d}\left(\sum_{i=1}^{d}\left[x_{i}\right]\right)$ on $H^{1}(X, \mathbb{R})$ given by the formula

$$
{ }_{p_{0}} \Phi_{d}\left(\sum_{i=1}^{d}\left[x_{i}\right]\right): c \in H^{1}(X, \mathbb{R}) \mapsto \sum_{i=1}^{d} \int_{p_{0}}^{x_{i}}\left(\omega_{c}+\bar{\omega}_{c}\right)
$$

(see formula $(2.14)$ for the definition of $\omega_{c}$ ).

If $c \in H^{1}(X, \mathbb{R})$ is the image of an integral cohomology class

$$
\gamma \in H^{1}(X, \mathbb{Z})
$$

we will write the corresponding holomorphic one-form $\omega_{c}$ as $\omega_{\gamma}$. 
Let ${ }_{p_{0}} \widetilde{f}_{d, \gamma}$ be the pull-back of the function ${ }_{d} \mathrm{e}^{2 \pi \mathrm{i} \varphi_{\gamma}}$ (see formula (2.19)) to $X^{(d)}$. Equivalently, ${ }_{p_{0}} \widetilde{f}_{d, \gamma}$ is the pull-back of the function $\mathrm{e}^{2 \pi \mathrm{i} \varphi_{\gamma}}$ under the map ${ }_{p_{0}} \Phi_{d}$. It follows from the definition of $p_{0} \Phi_{d}$ that the value of ${ }_{p_{0}} \widetilde{f}_{d, \gamma}$ at $\sum_{i=1}^{d}\left[x_{i}\right]$ is equal to

$$
\exp \left(2 \pi \mathrm{i}{ }_{p_{0}} \Phi_{d}\left(\sum_{i=1}^{d}\left[x_{i}\right]\right)(\gamma)\right)=\exp \left(2 \pi \mathrm{i} \sum_{i=1}^{d} \int_{p_{0}}^{x_{i}}\left(\omega_{\gamma}+\bar{\omega}_{\gamma}\right)\right)
$$

Thus, we obtain that ${ }_{p_{0}} \widetilde{f}_{d, \gamma}$ is given by the formula

$$
p_{0} \tilde{f}_{d, \gamma}\left(\sum_{i=1}^{d}\left[x_{i}\right]\right)=\exp \left(2 \pi \mathrm{i} \sum_{i=1}^{d} \int_{p_{0}}^{p}\left(\omega_{\gamma}+\bar{\omega}_{\gamma}\right)\right)=\prod_{i=1}^{d} \lambda_{x_{i}}^{\gamma},
$$

where

$$
\lambda_{p}^{\gamma}=\mathrm{e}^{2 \pi \mathrm{i} \int_{p_{0}}^{p}\left(\omega_{\gamma}+\bar{\omega}_{\gamma}\right)}
$$

We conclude that the functions ${ }_{p_{0}} \widetilde{f}_{d, \gamma}$ satisfy the factorization property (2.18). Therefore the function $\mathrm{e}^{2 \pi \mathrm{i} \varphi_{\gamma}}$ on $\operatorname{Pic}^{0}(X)$ is indeed an eigenfunction of ${ }_{p_{0}} H_{p}$, with the eigenvalue $\lambda_{p}^{\gamma}$ given by formula (2.22), which is what we wanted to prove. ${ }^{4}$

Thus, we have proved the following theorem.

Theorem 2.4. The joint eigenfunctions of the Hecke operators ${ }_{p_{0}} H_{p}, p \in X$, on $L^{2}\left(\operatorname{Pic}^{0}(X)\right)$ are the functions $\mathrm{e}^{2 \pi \mathrm{i} \varphi_{\gamma}}, \gamma \in H^{1}(X, \mathbb{Z})$. The eigenvalues of ${ }_{p_{0}} H_{p}$ are given by formula $(2.22)$, so that we have

$$
{ }_{p_{0}} H_{p} \cdot \mathrm{e}^{2 \pi \mathrm{i} \varphi_{\gamma}}=\mathrm{e}^{2 \pi \mathrm{i} \int_{p_{0}}^{p}\left(\omega_{\gamma}+\bar{\omega}_{\gamma}\right)} \mathrm{e}^{2 \pi \mathrm{i} \varphi_{\gamma}} .
$$

As in the case of an elliptic curve discussed in Section 2.2, the eigenvalues (2.22) can be interpreted as the holonomies of the flat unitary connections

$$
\nabla_{\gamma}=d-2 \pi \mathrm{i}\left(\omega_{\gamma}+\bar{\omega}_{\gamma}\right), \quad \gamma \in H^{1}(X, \mathbb{Z})
$$

on the trivial line bundle on $X$, taken along (no matter which) path from $p_{0}$ to $p$. As in the case of elliptic curves, the monodromy representation of each of these connections is trivial, ensuring that the Hecke eigenvalues $\lambda_{p}^{\gamma}$, viewed as functions of $p \in X$, are single-valued (see Section 2.3).

\subsection{General torus}

Let now $T$ be a connected torus over $\mathbb{C}$, and $\operatorname{Bun}_{T}(X)$ the moduli space of $T$-bundles on $X$ (note that the moduli stack $\operatorname{Bun}_{T}(X)$ is the quotient of $\operatorname{Bun}_{T}(X)$ by the trivial action of $T$ ). In Section 2.4 we find the joint eigenfunctions and eigenvalues of the Hecke operators in the case of $\operatorname{Bun}_{T}(X)$ where $T=\mathbb{G}_{m}$; in this case $\operatorname{Bun}_{\mathbb{G}_{m}}(X)=\operatorname{Pic}(X)$. Here we generalize these results to the case of an arbitrary $T$.

\footnotetext{
${ }^{4}$ Note that Abel's theorem implies that each function ${ }_{p_{0}} \widetilde{f}_{d, \gamma}, \gamma \in H^{1}(X, \mathbb{Z})$, is constant along the fibers of the Abel-Jacobi map $X^{(d)} \rightarrow$ Pic $^{d}$ and therefore descends to Pic ${ }^{d}$. This suggests another proof of Theorem 2.4: we start from the functions $p_{0} \widetilde{f}_{d, \gamma}$ on $X^{(d)}, d>0$. Formula $(2.21)$ shows that they combine into an eigenfunction of the operators $\widetilde{H}_{p}$. Hence the function on $\operatorname{Pic}^{d}(X), d \geq g$, to which ${ }_{p_{0}} \widetilde{f}_{d, \gamma}$ descends, viewed as a function on $\operatorname{Pic}^{0}(X)$ under the identification (2.17), is a Hecke eigenfunction. One can then show that this function is equal to $\mathrm{e}^{2 \pi \mathrm{i} \varphi_{\gamma}}$.
} 
Let $\Lambda^{*}(T)$ and $\Lambda_{*}(T)$ be the lattices of characters and cocharacters of $T$, respectively. Any $\mathcal{P} \in \operatorname{Bun}_{T}(X)$ is uniquely determined by the $\mathbb{G}_{m}$-bundles (equivalently, line bundles) $\mathcal{P} \underset{\mathbb{G}_{m}}{\times} \chi$ associated to the characters $\chi: T \rightarrow \mathbb{G}_{m}$ in $\Lambda^{*}(T)$. This yields a canonical isomorphism

$$
\operatorname{Bun}_{T}(X) \simeq \operatorname{Pic}(X) \underset{\mathbb{Z}}{\otimes} \Lambda_{*}(T)=\bigsqcup_{\check{\nu} \in \Lambda_{*}(T)} \operatorname{Bun}_{T}^{\check{L}}(X)
$$

The neutral component

$$
\operatorname{Bun}_{T}^{0}(X)=\operatorname{Pic}^{0}(X) \underset{\mathbb{Z}}{\otimes} \Lambda_{*}(T)
$$

is non-canonically isomorphic to $\operatorname{Pic}^{0}(X)^{r}$, where $r$ is the rank of the lattice $\Lambda_{*}(T)$.

The Hecke operators $H_{p}^{\check{\mu}}$ are now labeled by $p \in X$ and $\check{\mu} \in \Lambda_{*}(T)$. The operator $H_{p}^{\check{\mu}}$ corresponds to the pull-back under the map

$$
\begin{aligned}
T_{p}^{\check{\mu}}: \operatorname{Bun}_{T}^{\check{\nu}}(X) & \rightarrow \operatorname{Bun}_{T}^{\check{\nu}+\check{\mu}}(X), \\
\mathcal{P} & \mapsto \mathcal{P}(\check{\mu} \cdot p),
\end{aligned}
$$

where $\mathcal{P}(\check{\mu} \cdot p)$ is defined by the formula

$$
\mathcal{P}(\check{\mu} \cdot p) \underset{\mathbb{G}_{m}}{\times} \chi=\left(\mathcal{P} \underset{\mathbb{G}_{m}}{\times} \chi\right)(\langle\chi, \check{\mu}\rangle \cdot p), \quad \chi \in \Lambda^{*}(T) .
$$

As in the case of $T=\mathbb{G}_{m}$, we choose, once and for all, a reference point $p_{0} \in X$.

As in the case of $T=\mathbb{G}_{m}$, finding eigenfunctions and eigenvalues of the commuting operators $H_{p}^{\breve{\mu}}$ on functions on $\operatorname{Bun}_{T}(X)$ is equivalent to finding eigenfunctions and eigenvalues of the operators

$$
{ }_{p_{0}} H_{p}^{\check{\mu}}=\left(H_{p_{0}}^{\check{\mu}}\right)^{-1} \circ H_{p}^{\check{\mu}}
$$

on functions on $\operatorname{Bun}_{T}^{0}(X)$. As in the case of $T=\mathbb{G}_{m}$, we represent $\operatorname{Bun}_{T}^{0}(X)$ as

$$
\operatorname{Bun}_{T}^{0}(X) \simeq H^{1}\left(X, \mathfrak{t}_{\mathbb{R}}^{*}\right)^{*} / H_{1}\left(X, \Lambda_{*}(T)\right),
$$

where $\mathfrak{t}_{\mathbb{R}}=\mathbb{R} \underset{\mathbb{Z}}{\times} \Lambda_{*}(T)$ is the split real form of the complex Lie algebra $\mathfrak{t}$ of $T$.

As in Section 2.4, for any

$$
\gamma \in H^{1}\left(X, \Lambda^{*}(T)\right)
$$

the image of $\gamma$ in $H^{1}\left(X, \mathfrak{t}_{\mathbb{R}}^{*}\right)$ is represented by a unique $\mathfrak{t}_{\mathbb{R}}^{*}$-valued one-form on $X$ that may be written as

$$
\omega_{\gamma}+\bar{\omega}_{\gamma}
$$

where $\omega_{\gamma} \in H^{0}\left(X, \Omega^{1,0}\right) \underset{\mathbb{C}}{\otimes} \mathfrak{t}^{*}$ is a holomorphic $\mathfrak{t}^{*}$-valued one-form.

On the other hand, the image of $\gamma$ in $H^{1}\left(X, \mathfrak{t}_{\mathbb{R}}^{*}\right)$ gives rise to a linear functional

$$
\varphi_{\gamma}: H^{1}\left(X, \mathfrak{t}_{\mathbb{R}}^{*}\right)^{*} \rightarrow \mathbb{R}
$$

satisfying $\varphi_{\gamma}(\beta) \in \mathbb{Z}$ for all $\beta \in H_{1}\left(X, \Lambda_{*}(T)\right)$. Therefore, according to formula (2.23), $\mathrm{e}^{2 \pi \mathrm{i} \varphi_{\gamma}}$ is a well-defined function on $\operatorname{Bun}_{T}^{0}(X)$. These are the Fourier harmonics on $\operatorname{Bun}_{T}^{0}(X)$.

In the same way as in Section 2.4, we prove the following result. 
Theorem 2.5. The functions $\mathrm{e}^{2 \pi \mathrm{i} \varphi_{\gamma}}, \gamma \in H^{1}\left(X, \Lambda^{*}(T)\right)$, form an orthogonal basis of joint eigenfunctions of the Hecke operators $p_{0} H_{p}^{\breve{\mu}}, p \in X, \check{\mu} \in \Lambda_{*}(T)$, on $L^{2}\left(\operatorname{Bun}_{T}^{0}(X)\right)$. The eigenvalues of ${ }_{p_{0}} H_{p}^{\check{\mu}}$ are given by the right hand side of the formula

$$
{ }_{p_{0}} H_{p}^{\check{\mu}} \cdot \mathrm{e}^{2 \pi \mathrm{i} \varphi_{\gamma}}=\check{\mu}\left(\mathrm{e}^{2 \pi \mathrm{i} \int_{p_{0}}^{p}\left(\omega_{\gamma}+\bar{\omega}_{\gamma}\right)}\right) \mathrm{e}^{2 \pi \mathrm{i} \varphi_{\gamma}} .
$$

Let us explain the notation we used on the right hand side of formula (2.24): denote by ${ }^{L} T$ the Langlands dual torus to $T$. We have $\Lambda_{*}\left({ }^{L} T\right)=\Lambda^{*}(T)$ and $\Lambda^{*}\left({ }^{L} T\right)=\Lambda_{*}(T)$. The eigenvalue of the Hecke operator ${ }_{p_{0}} H_{p}^{\breve{\mu}}, p \in X, \check{\mu} \in \Lambda^{*}\left({ }^{L} T\right)$, on the function $\mathrm{e}^{2 \pi \mathrm{i} \varphi_{\gamma}}$ is equal to the value of the character $\check{\mu}$ of ${ }^{L} T$ on the ${ }^{L} T$-valued function $F_{\gamma}$ on $X$

$$
F_{\gamma}(p)=\mathrm{e}^{2 \pi \mathrm{i} \int_{p_{0}}^{p}\left(\omega_{\gamma}+\bar{\omega}_{\gamma}\right)}, \quad \gamma \in H^{1}\left(X, \Lambda^{*}(T)\right)=H^{1}\left(X, \Lambda_{*}\left({ }^{L} T\right)\right) .
$$

This function actually takes values in the compact form ${ }^{L} T_{u}$ of ${ }^{L} T$ and may be interpreted as the holonomy of the unitary connection

$$
\nabla_{\gamma}=d-2 \pi \mathrm{i}\left(\omega_{\gamma}+\bar{\omega}_{\gamma}\right)
$$

on the trivial ${ }^{L} T_{u}$-bundle on $X$ over (no matter which) path from $p_{0}$ to $p$. As in the case of $T=\mathbb{G}_{m}$, each of these connections has trivial monodromy.

\section{Non-abelian case}

In this section we try to generalize to the case of a non-abelian group $G$ the results obtained in the previous section for abelian $G$.

\subsection{Spherical Hecke algebra for groups over $\mathbb{F}_{q}((t))$}

In the case of the function field of a curve $X$ over a finite field, the Hecke operators attached to a closed point $x$ of $X$ generate the spherical Hecke algebra $\mathcal{H}\left(G\left(\mathbb{F}_{q}((t))\right), G\left(\mathbb{F}_{q}[[t]]\right)\right)$. As a vector space, it is the space of $\mathbb{C}$-valued functions on the group $G\left(\mathbb{F}_{q}((t))\right)$ that are bi-invariant with respect to the subgroup $G\left(\mathbb{F}_{q}[[t]]\right)$ (here $\mathbb{F}_{q}$ is the residue field of $x$ ). This vector space is endowed with the convolution product defined by the formula

$$
\left(f_{1} \star f_{2}\right)(g)=\int f_{1}\left(g h^{-1}\right) f_{2}(h) \mathrm{d} h,
$$

where $\mathrm{d} h$ stands for the Haar measure on $G\left(\mathbb{F}_{q}((t))\right)$ normalized so that the volume of the subgroup $G\left(\mathbb{F}_{q}[[t]]\right)$ is equal to 1 (in this normalization, the characteristic function of $G\left(\mathbb{F}_{q}[[t]]\right)$ is the unit element of the convolution algebra). The Haar measure can be defined because $G\left(\mathbb{F}_{q}((t))\right)$ is a locally compact group.

The resulting convolution algebra $\mathcal{H}\left(G\left(\mathbb{F}_{q}((t))\right), G\left(\mathbb{F}_{q}[[t]]\right)\right)$ is commutative and we have the Satake isomorphism between this algebra and the complexified representation $\operatorname{ring} \operatorname{Rep}^{L} G$ of the Langlands dual group ${ }^{L} G$.

\subsection{Is there a spherical Hecke algebra for groups over $\mathbb{C}((t))$ ?}

In contrast to the group $G\left(\mathbb{F}_{q}((t))\right)$, the group $G(\mathbb{C}((t)))$ is not locally compact. Therefore it does not carry a Haar measure. Indeed, the field $\mathbb{C}((t))$ is an example of a two-dimensional local field, in the terminology of [19], more akin to $\mathbb{F}_{q}((z))((t))$ or $\mathbb{Q}_{p}((t))$ than to $\mathbb{F}_{q}((t))$ or $\mathbb{Q}_{p}$.

Ivan Fesenko has developed integration theory for the two-dimensional local fields [19, 20], and his students have extended it to algebraic groups over such fields [48, 49, 52], but this theory is quite different from the familiar case of $G\left(\mathbb{F}_{q}((t))\right)$. 
First, integrals over $\mathbb{C}((t))$ and $G(\mathbb{C}((t)))$ take values not in real numbers, but in formal Laurent power series $\mathbb{R}((X))$, where $X$ is a formal variable. Under certain restrictions, the value of the integral is a polynomial in $X$; if so, then one could set $X$ to be equal to a real number. But this way one might lose some important properties of the integration that we normally take for granted.

Second, if $S$ is a Lebesque measurable subset of $\mathbb{C}$, then according to [19, 20], the measure of a subset of $\mathbb{C}((t))$ of the form

$$
S t^{i}+t^{i+1} \mathbb{C}[[t]],
$$

is equal to $\mu(S) X^{i}$, where $\mu(S)$ is the usual Lebesque measure of $S$. In particular, this means that the measure of the subset $\mathbb{C}[[t]]$ of $\mathbb{C}((t))$ is equal to 0 , as is the measure of the subset $t^{n} \mathbb{C}[[t]]$ for any $n \in \mathbb{Z}$. Contrast this with the fact that under a suitably normalized Haar measure on $\mathbb{F}_{q}((t))$, the measure of $t^{n} \mathbb{F}_{q}[[t]]$ is equal to $q^{-n}$. Thus, if we take as $G$ the additive group, it's not even clear how to define a unit element in the would-be spherical Hecke algebra (which would be the characteristic function of the subset $\mathbb{F}_{q}[[t]]$ in the case of the field $\left.\mathbb{F}_{q}((t))\right)$. The situation is similar in the case of a general group $G$.

For this reason, according to Waller [51], from the point of view of the two-dimensional integration theory it would make more sense to consider distributions on $G((t))$ that are biinvariant not with respect to $G(\mathbb{C}[[t]])$, but its subgroup $\widetilde{K}$ consisting of those elements $g(t) \in$ $G(\mathbb{C}[[t]])$ for which $g(0)$ belongs to a compact subgroup $K$ of $G(\mathbb{C})$. This would be similar to the construction used in representation theory of complex Lie groups, where one considers, for example, the space of distributions on the group $G(\mathbb{C})$ supported on a compact subgroup $K$ with a natural convolution product [38]. ${ }^{5}$ For instance, if $K=\{1\}$, the resulting algebra is $U(\mathfrak{g})$, the universal enveloping algebra of the Lie algebra $\mathfrak{g}$ of $G(\mathbb{C})$ (see also Remark 4.1 below.)

Perhaps, a convolution product on some space of distributions of this kind can be defined for $G(\mathbb{C}((t)))$, but from the structure of the double cosets of $\widetilde{K}$ in $G(\mathbb{C}((t)))$ it is clear that this algebra would not be pertinent to defining Hecke operators on $\operatorname{Bun}_{G}$.

Another option is to consider motivic integration theory. A motivic version of the Haar measure has in fact been defined by Julia Gordon [32] for the group $G(\mathbb{C}((t))$ ) (it may also be obtained in the framework of the general theories of Cluckers-Loeser [11] or HrushovskiKazhdan [34]). Also, in a recent paper [10] it was shown that the spherical Hecke algebra $\mathcal{H}\left(G\left(\mathbb{F}_{q}((t))\right), G\left(\mathbb{F}_{q}[[t]]\right)\right)$ can be obtained by a certain specialization from its version in which the ordinary integration with respect to the Haar measure on $G\left(\mathbb{F}_{q}((t))\right)$ is replaced by the motivic integration with respect to the motivic Haar measure. Presumably, one could carry some of the results of $[10]$ over to the case of $\mathbb{C}((t))$.

However, this does not seem to give us much help, for the following reason: the motivic integrals over a ground field $k$ take values in a certain algebra $\mathcal{M}_{k}$, which is roughly speaking a localization of the Grothendieck ring of algebraic varieties over $k$. In the case of the ground field $\mathbb{F}_{q}$, the algebra $\mathcal{M}_{\mathbb{F}_{q}}$ is rich, and ordinary integrals may be recovered from the motivic ones by taking a homomorphism from $\mathcal{M}_{\mathbb{F}_{q}}$ to $\mathbb{R}$ sending the class of the affine line over $\mathbb{F}_{q}$ to $q$. But in the case of the ground field $\mathbb{C}$, the structure of the algebra $\mathcal{M}_{\mathbb{C}}$ appears to be very different (for example, it has divisors of zero), and this construction does not work. In fact, it seems that there are very few (if any) known homomorphisms from $\mathcal{M}_{\mathbb{C}}$ to positive real numbers, besides the Euler characteristic. ${ }^{6}$ Perhaps, taking the Euler characteristic, one can obtain a non-trivial convolution algebra structure on the space of $G(\mathbb{C}[[t]])$ bi-invariant functions on $G(((t))$ ) (one may wonder whether it could be interpreted as a kind of $q \rightarrow 1$ limit of $\left.\mathcal{H}\left(G\left(\mathbb{F}_{q}((t))\right), G\left(\mathbb{F}_{q}[[t]]\right)\right)\right)$, but it is doubtful that this algebra could be useful in any way for defining an analytic theory of automorphic forms on $\operatorname{Bun}_{G}$ for complex algebraic curves.

\footnotetext{
${ }^{5}$ I thank David Vogan for telling me about this construction, and the reference.

${ }^{6}$ I learned this from David Kazhdan (private communication).
} 


\subsection{An attempt to define Hecke operators}

What we are interested in here, however, is not the spherical Hecke algebra itself, but rather the action of the corresponding Hecke operators on automorphic functions. After all, in Section 2 we were able to define Hecke operators without any reference to a convolution algebra on the group $\mathbb{C}((t))^{\times}$. The abelian case, however, is an exception in that the action of the Hecke operators did not require integration. In the non-abelian case (in fact, already for $G=\mathrm{GL}_{2}$ ), integration is necessary, and this presents various difficulties, which I illustrate below with some concrete examples in the case of $\mathrm{GL}_{2}$ and an elliptic curve.

Recall that for curves over $\mathbb{F}_{q}$, the unramified automorphic functions are functions on the double quotient

$$
G(F) \backslash G\left(\mathbb{A}_{F}\right) / G\left(\mathcal{O}_{F}\right),
$$

where $F=\mathbb{F}_{q}(X)$, and $X$ is a curve over $\mathbb{F}_{q}$. The action of Hecke operators on functions on this double quotient can be defined by means of certain correspondences, and we can try to imitate this definition for complex curves.

To this end, we take the same double quotient (3.1) with $F=\mathbb{C}(X)$, where $X$ is a curve over $\mathbb{C}$. As in the case of $\mathbb{F}_{q}$, this is the set of equivalence classes of principle $G$-bundles on $X$. The Hecke correspondences can be conveniently defined in these terms.

For instance, consider the case of $\mathrm{GL}_{2}$ and the first Hecke operator (for a survey of the general case, see, e.g., [23, Section 3.7]). Then we have the Hecke correspondence $\mathcal{H e c k e}_{1, x}$, where $x$ is a closed point of $X$ :

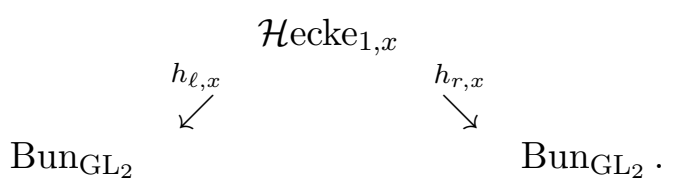

Here $\mathcal{H}$ ecke $_{1, x}$ is the moduli stack classifying the quadruples

$$
\left(\mathcal{M}, \mathcal{M}^{\prime}, \beta: \mathcal{M}^{\prime} \hookrightarrow \mathcal{M}\right),
$$

where $\mathcal{M}$ and $\mathcal{M}^{\prime}$ are points of $\mathrm{Bun}_{\mathrm{GL}_{2}}$, which means that they are rank two vector bundles on $X$, and $\beta$ is an embedding of their sheaves of (holomorphic) sections $\beta: \mathcal{M}^{\prime} \hookrightarrow \mathcal{M}$ such that $\mathcal{M} / \mathcal{M}^{\prime}$ is supported at $x$ and is isomorphic to the skyscraper sheaf $\mathcal{O}_{x}=\mathcal{O}_{X} / \mathcal{O}_{X}(-x)$. The maps are defined by the formulas $h_{\ell, x}\left(\mathcal{M}, \mathcal{M}^{\prime}, \beta\right)=\mathcal{M}, h_{r, x}\left(\mathcal{M}, \mathcal{M}^{\prime}, \beta\right)=\mathcal{M}^{\prime}$.

It follows that the points of the fiber of $\mathcal{H e c k e}_{1, x}$ over $\mathcal{M}$ in the "left" $\mathrm{Bun}_{\mathrm{GL}_{2}}$ correspond to all locally free subsheaves $\mathcal{M}^{\prime} \subset \mathcal{M}$ such that the quotient $\mathcal{M} / \mathcal{M}^{\prime}$ is the skyscraper sheaf $\mathcal{O}_{x}$. Defining such $\mathcal{M}^{\prime}$ is the same as choosing a line $L$ in the dual space $\mathcal{M}_{x}^{*}$ to the fiber of $\mathcal{M}$ at $x$ (which is a two-dimensional complex vector space). The sections of the corresponding sheaf $\mathcal{M}^{\prime}$ (over a Zariski open subset of $X$ ) are the sections of $\mathcal{M}$ that vanish along $L$, i.e., sections $s$ which satisfy the equation $\langle v, s(x)\rangle=0$ for a non-zero $v \in L$.

Thus, the fiber of $\mathcal{H}$ ecke $_{1, x}$ over $\mathcal{M}$ is isomorphic to the projectivization of the two-dimensional vector space $\mathcal{M}_{x}^{*}$, i.e., to $\mathbb{C P}^{1}$. We conclude that $\mathcal{H}$ ecke $e_{1, x}$ is a $\mathbb{C P}^{1}$-fibration over the "left" Bun $_{\mathrm{GL}_{2}}$ in the diagram (3.2). Likewise, we obtain that $\mathcal{H}$ ecke $_{1, x}$ is a $\mathbb{C P}^{1}$-fibration over the "right" Bun $_{\mathrm{GL}_{2}}$ in (3.2).

In the geometric theory, we use the correspondence (3.2) to define a Hecke functor $\mathrm{H}_{1, x}$ on the (derived) category of $D$-modules on $\mathrm{Bun}_{\mathrm{GL}_{2}}$ :

$$
\mathrm{H}_{1, x}(\mathcal{K})=h_{\ell, x *} h_{r, x}^{*}(\mathcal{K})[1] .
$$

A $D$-module $\mathcal{K}$ is called a Hecke eigensheaf if we have isomorphisms

$$
\imath_{1, x}: \quad \mathrm{H}_{1, x}(\mathcal{K}) \stackrel{\sim}{\longrightarrow} \mathbb{C}^{2} \otimes \mathcal{K} \simeq \mathcal{K} \oplus \mathcal{K}, \quad \forall x \in X,
$$


and in addition have similar isomorphisms for the second set of Hecke functors $\mathrm{H}_{2, x}, x \in X$. These are defined similarly to the Hecke operators for $\mathrm{GL}_{1}$, as the pull-backs with respect to the morphisms sending a rank two bundle $\mathcal{M}$ to $\mathcal{M}(x)$ (they change the degree of $\mathcal{M}$ by 2). (As explained in [25, Section 1.1], the second Hecke eigensheaf property follows from the first together with a certain $S_{2}$-equivariance condition.)

Thus, if $\mathcal{K}$ is a Hecke eigensheaf, we obtain a family of isomorphisms (3.3) for all $x \in X$, and similarly for the second set of Hecke functors. We then impose a stronger requirement that the two-dimensional vector spaces appearing on the right hand side of (3.3) as "eigenvalues" fit together as stalks of a single rank two local system $\mathcal{E}$ on $X$ (and similarly for the second set of Hecke functors, where the eigenvalues should be the stalks of the rank one local system $\wedge^{2} \mathcal{E}$ on $X$; this is, however, automatic if we impose the $S_{2}$-equivariance condition from [25, Section 1.1]). If that's the case, we say that $\mathcal{K}$ is a Hecke eigensheaf with the eigenvalue $\mathcal{E}$. This is explained in more detail, e.g., in [23, Section 3.8].

The first task of the geometric theory (in the case of $G=\mathrm{GL}_{2}$ ) is to show that such a Hecke eigensheaf on Bun $_{\mathrm{GL}_{2}}$ exists for every irreducible rank two local system $\mathcal{E}$ on $X$. This was accomplished by Drinfeld in [13], a groundbreaking work that was the starting point of the geometric theory. We now know that the same is true for $G=\mathrm{GL}_{n}[25,26]$ and in many other cases.

Now let's try to adapt the diagram (3.2) to functions. Thus, given a function $f$ on the set of $\mathbb{C}$-points, we wish to define the action of the first Hecke operator $H_{1, x}$ on it by the formula

$$
\left(H_{1, x} \cdot f\right)(\mathcal{M})=\int_{\mathcal{M}^{\prime} \in h_{\ell, x}^{-1}(\mathcal{M})} f\left(\mathcal{M}^{\prime}\right) \mathrm{d} \mathcal{M}^{\prime}
$$

Thus, we see that the result must be an integral over the complex projective line $h_{\ell, x}^{-1}(\mathcal{M})$. The key question is: what is the measure $\mathrm{d} \mathcal{M}^{\prime}$ ?

Herein lies a crucial difference with the abelian case considered in Section 2: in the abelian case every Hecke operator acted by pull-back of a function, so no integration was needed. But in the non-abelian case, already for the first Hecke operators $H_{1, x}$ in the case of $G=G_{2}$, we must integrate functions over the projective lines $h_{\ell, x}^{-1}(\mathcal{M})$, where $\mathcal{M} \in \operatorname{Bun}_{\mathrm{GL}_{2}}(\mathbb{C})$.

Note that if our curve were over a finite field, this integration is in fact a summation over a finite set of $q+1$ elements, the number of points of $\mathbb{P}^{1}$ over $\mathbb{F}_{q}$, where $\mathbb{F}_{q}$ is the residue field of the closed point $x$ at which we take the Hecke operator. The terms of this summation correspond to points of the fibers $h_{\ell, x}^{-1}(\mathcal{M})$. Being finite sums, these integrals are always well-defined if our curve is over $\mathbb{F}_{q}$. For curves over $\mathbb{C}$, this is not so, and this creates major problems, as we will see below.

\subsection{The case of an elliptic curve}

Let's look at the case of an elliptic curve $X$. If it is defined over a finite field, the fibers $h_{\ell, x}^{-1}(\mathcal{M})$ appearing in the Hecke operators have been described explicitly in $[1,45]$, using the classification of rank two bundles on elliptic curves due to Atiyah [3].

For a complex elliptic curve $X$, the fibers $h_{\ell, x}^{-1}(\mathcal{M})$ have been described explicitly in [7]. In [42], Langlands attempts to describe them in the language of adeles, which is more unwieldy than the vector bundle language used in [7] and hence more prone to errors. As the result of his computations, Langlands states on p. 18 of [42]:

"The dimension $\operatorname{dim}\left(g \Delta_{1} / G\left(\mathcal{O}_{x}\right)\right)$ [which is our $h_{\ell, x}^{-1}(\mathcal{M})$ if $\mathcal{M}$ is the rank two bundle corresponding to the adele $g \in \mathrm{GL}_{2}\left(\mathbb{A}_{F}\right)$ ] is always equal to $0 \ldots$ Hence the domain of integration in [adelic version of our formula (3.4) above] is a finite set". 
This statement is incorrect. First of all, as we show below, there are rank two bundles $\mathcal{M}$ on an elliptic curve for which there are infinitely many non-isomorphic bundles in the fiber $h_{\ell, x}^{-1}(\mathcal{M})$ (in fact, we have a continuous family of non-isomorphic bundles parametrized by the points of $h_{\ell, x}^{-1}(\mathcal{M})$ ). Second, even if there are finitely many isomorphism classes among those $\mathcal{M}^{\prime}$ which appear in the fiber $h_{\ell, x}^{-1}(\mathcal{M})$ for a fixed $\mathcal{M}$, this does not mean that we are integrating over a finite set.

In fact, according to formula (3.4) (whose adelic version is formula (10) of [42]), for every $\mathcal{M}$, the fiber $h_{\ell, x}^{-1}(\mathcal{M})$ of the Hecke correspondence over which we are supposed to integrate is always isomorphic to $\mathbb{C P}^{1}$ if we take into account the automorphism groups of the bundles involved. In the adelic language, the automorphism group $\operatorname{Aut}(\mathcal{M})$ of a bundle $\mathcal{M}$ may be described, up to an isomorphism, as follows: $\mathcal{M}$ corresponds to a point in the double quotient (3.1); we lift this point to $G(F) \backslash G\left(\mathbb{A}_{F}\right)$ and take its stabilizer subgroup in $G\left(\mathcal{O}_{F}\right)$.

The necessity to take into account these automorphism groups is well-know in the case of curves defined over $\mathbb{F}_{q}$. In this case, the measure on the double quotient (3.1) induced by the Tamagawa measure assigns (up to an overall factor) to a point not 1 but $1 /|\operatorname{Aut}(\mathcal{M})|$ (this measure is well-defined if we work over $\mathbb{F}_{q}$ because then the group $\operatorname{Aut}(\mathcal{M})$ is finite for any $\mathcal{M}$; however, this is not so over $\mathbb{C})$. With respect to this correctly defined measure, the fiber $h_{\ell, x}^{-1}(\mathcal{M})$ for any $\mathcal{M}$ and any $\mathbb{F}_{q}$-point $x$ of $X$ can be identified with the set of $\mathbb{F}_{q}$-points of the projective line over $\mathbb{F}_{q}$, with each point having measure 1 .

As a concrete illustration, consider the following example.

Example 3.1. Let $\mathcal{M}=\mathcal{L}_{1} \oplus \mathcal{L}_{2}$, where $\mathcal{L}_{1}$ and $\mathcal{L}_{2}$ are two line bundles of degrees $d_{1}$ and $d_{2}$ such that $d_{1}>d_{2}+1$. Then the vector bundles $\mathcal{M}^{\prime}$ that appear in the fiber $h_{\ell, x}^{-1}(\mathcal{M})$ are isomorphic to either $\mathcal{M}_{1}^{\prime}=\mathcal{L}_{1}(-x) \oplus \mathcal{L}_{2}$ or $\mathcal{M}_{2}^{\prime}=\mathcal{L}_{1} \oplus \mathcal{L}_{2}(-x)$. However, the groups of automorphisms of these bundles are different: each of them is a semi-direct product of the group $\left(\mathbb{C}^{\times}\right)^{2}$ of rescalings of the two line bundles appearing in a direct sum decomposition and an additive group, which is $\operatorname{Hom}\left(\mathcal{L}_{2}, \mathcal{L}_{1}\right)$ for $\mathcal{M} ; \operatorname{Hom}\left(\mathcal{L}_{2}, \mathcal{L}_{1}(-x)\right)$ for $\mathcal{M}_{1}^{\prime}$; and $\operatorname{Hom}\left(\mathcal{L}_{2}(-x), \mathcal{L}_{1}\right)$ for $\mathcal{M}_{2}^{\prime}$.

Under our assumption that $d_{1}>d_{2}+1$, we find that the latter groups are isomorphic to $\mathbb{C}^{d_{1}-d_{2}-1}, \mathbb{C}^{d_{1}-d_{2}-2}$, and $\mathbb{C}^{d_{1}-d_{2}}$, respectively. Thus, the automorphism group of $\mathcal{M}_{1}^{\prime}$ is "smaller" by one copy of the additive group $\mathbb{G}_{a}$ than that of $\mathcal{M}$, whereas the automorphism group of $\mathcal{M}_{2}^{\prime}$ is larger than that of $\mathcal{M}$ by the same amount.

This implies that the fiber $h_{\ell, x}^{-1}(\mathcal{M})$ is the union of a complex affine line worth of points corresponding to $\mathcal{M}_{1}^{\prime}$ and a single point corresponding to $\mathcal{M}_{2}^{\prime}$.

If we worked over $\mathbb{F}_{q}$, we would find that

$$
\left(H_{1, x} \cdot f\right)(\mathcal{M})=q f\left(\mathcal{M}_{1}^{\prime}\right)+f\left(\mathcal{M}_{2}^{\prime}\right),
$$

with the factor of $q$ representing the number of points of the affine line (see $[1,45])$. Over $\mathbb{C}$, we formally obtain the sum of two terms: (1) an integral of the constant function taking value $f\left(\mathcal{M}_{1}^{\prime}\right)$ on an open dense subset of $\mathbb{C P}^{1}$ isomorphic to the affine line, and (2) a single term $f\left(\mathcal{M}_{2}^{\prime}\right)$ corresponding to the remaining point. Is there an integration measure of $\mathbb{C P}^{1}$ that would render this sum meaningful?

If we use a standard integration measure on $\mathbb{C P}^{1}$, then the answer would be $f\left(\mathcal{M}_{1}^{\prime}\right)$ multiplied by the measure of the affine line. The second term would drop out, as it would correspond to a subset (namely, a point) of measure zero. If we want to include the second bundle (which we certainly do for Hecke operators to be meaningful), then the measure of this point has to be non-zero. But we also expect our measure on $\mathbb{C P}^{1}$ to be invariant (indeed, we cannot a priori distinguish a special point on each of these projective lines). Therefore the measure of every point of $\mathbb{C P}^{1}$ would have to be given by the same non-zero number. But then our integral would diverge. It is not clear how one could regularize these divergent integrals in a uniform and meaningful way. 
Next, we give an example in which the fiber $h_{\ell, x}^{-1}(\mathcal{M})$ is a continuous family of non-isomorphic vector bundles (thus directly contradicting the above statement from [42]).

Example 3.2. Let $\mathcal{M}$ be the indecomposable rank two degree 1 vector bundle $F_{2}(x)$ (in the notation of [42]) which is a unique, up to an isomorphism, non-trivial extension

$$
0 \rightarrow \mathcal{O}_{X} \rightarrow F_{2}(x) \rightarrow \mathcal{O}_{X}(x) \rightarrow 0 .
$$

In this case, as shown in [7, Section 4.3], the fiber $h_{\ell, x}^{-1}\left(F_{2}(x)\right)$ may be described in terms of a canonical two-sheeted covering $\pi: \operatorname{Pic}^{0}(X) \rightarrow \mathbb{C P}^{1}=h_{\ell, x}^{-1}\left(F_{2}(x)\right)$ ramified at 4 points such that (1) if $a \in h_{\ell, x}^{-1}\left(F_{2}(x)\right)$ is outside of the ramification locus, then $\pi^{-1}(a)=\left\{\mathcal{L}[a], \mathcal{L}[a]^{-1}\right\}$, where $\mathcal{L}[a]$ is a degree 0 line bundle on $X$; and (2) the fibers over the 4 ramification points are the four square roots $\mathcal{L}_{i}, i=1, \ldots, 4$, of the trivial line bundle on $X$.

Namely, the vector bundle $\mathcal{M}^{\prime}(a)$ corresponding to a point $a \in h_{\ell, x}^{-1}\left(F_{2}(x)\right)$ is described in terms of $\pi$ as follows (note that in [7] the bundle $F_{2}(x)$ is denoted by $G_{2}(x)$ and $\mathcal{L}[a]$ is denoted by $\mathcal{L}(a))$ :

- if $a \in h_{\ell, x}^{-1}\left(F_{2}(x)\right)$ is outside of the ramification locus, then $\mathcal{M}^{\prime}(a)=\mathcal{L}[a] \oplus \mathcal{L}[a]^{-1}$;

- if $a$ is a ramification point corresponding to the line bundle $\mathcal{L}_{i}$, then $\mathcal{M}^{\prime}(a)=\mathcal{L}_{i} \otimes F_{2}$, where $F_{2}$ is the unique, up to an isomorphism, non-trivial extension of $\mathcal{O}_{X}$ by itself.

According to the Atiyah's classification, the bundles $\mathcal{M}^{\prime}(a)$ and $\mathcal{M}^{\prime}(b)$ corresponding to different points $a \neq b$ in $h_{\ell, x}^{-1}\left(F_{2}(x)\right)$ are non-isomorphic. Thus, there is an infinite continuous family of non-isomorphic vector bundles appearing in the fiber $h_{\ell, x}^{-1}\left(F_{2}(x)\right)$ in this case.

One gets a similar answer for $\mathcal{M}=F_{2}(x) \otimes \mathcal{L}$, where $\mathcal{L}$ is an arbitrary line bundle on $X$ (note that unlike the vector bundles discussed in the previous example, all of the bundles $F_{2}(x) \otimes \mathcal{L}$ are stable). This means that the value of $H_{1, x} \cdot f$ at bundles $\mathcal{M}$ of this form depends on the choice of a measure of integration on $h_{\ell, x}^{-1}(\mathcal{M})$.

It is not clear whether it is possible to define these measures for different $\mathcal{M}$ and different $x \in X$ in a consistent and meaningful way, so that they would not only yield well-defined integrals but that the corresponding operators $H_{1, x}, x \in X$, would commute with each other and with the second set of Hecke operators $H_{2, x}, x \in X$.

In [42], Langlands sidesteps these problems and instead defines his versions of the Hecke operators by explicit formulas. Let $D$ (resp. $U$ ) be the substacks of Bun $\mathrm{GL}_{2}$ of an elliptic curve parametrizing rank two vector bundles on an elliptic curve $X$ that are decomposable (resp. indecomposable) as direct sums of line bundles. Points in both substacks can be explicitly described using Atiyah's classification results [3]. If we forget the automorphism groups of these rank two bundles, we obtain algebraic varieties $\mathfrak{D}$ and $\mathfrak{U}$, the former isomorphic to $\operatorname{Sym}^{2}(\operatorname{Pic}(X))$ and the latter isomorphic to a disjoint union of $\operatorname{Pic}(X)$ and $\operatorname{Pic}(X) / \operatorname{Pic}_{2}(X)$, where $\operatorname{Pic}_{2}(X)$ is the subgroup of line bundles $\mathcal{L}$ such that $\mathcal{L}^{\otimes 2} \simeq \mathcal{O}_{X}$ (they correspond to the indecomposable bundles of even and odd degrees, respectively).

In [42, pp. 20-21], Langlands defines his versions of the Hecke operators as linear operators acting on the direct sum $L^{2}(\mathfrak{D}) \oplus L^{2}(\mathfrak{U})$. He postulates (as he writes on p. 21, "by decree!") that these Hecke operators should act on this space diagonally, i.e., preserving each of the two subspaces $L^{2}(\mathfrak{D})$ and $L^{2}(\mathfrak{U})$.

However, the idea of treating the moduli stack of rank two bundles on $X$ as the disjoint union of the varieties $\mathfrak{D}$ and $\mathfrak{U}$ sounds problematic. Indeed, in the moduli stack $\mathrm{Bun}_{\mathrm{GL}_{2}}$ the substacks $D$ and $U$ are "glued" together in a non-trivial way. ${ }^{7}$ If we tear them apart, we are at

\footnotetext{
${ }^{7}$ Note that if we were to consider instead the moduli space of semi-stable bundles, then, depending on the stability condition we choose, some of the decomposable bundles in $\mathfrak{D}$ would have to be removed, or identified with the indecomposable ones in $\mathfrak{U}$. Considering the moduli space of semi-stable bundles is, however, problematic for a different reason: it is not preserved by the Hecke correspondences.
} 
the same time tearing apart the projective lines $h_{\ell, x}^{-1}(\mathcal{M})$ appearing as the fibers of the Hecke correspondences. The idea of defining the Hecke operators in such away that we keep the part corresponding to the bundles of one type and throw away the part corresponding to the bundles of the other type sounds even more problematic, for the following reason.

Consider the stable indecomposable bundle $F_{2}(x)$ described in Example 3.2 above. It corresponds to a point of $\mathfrak{U}$. But, as explained in Example 3.2, all but 4 points in the Hecke fiber $h_{\ell, x}^{-1}\left(F_{2}(x)\right) \simeq \mathbb{C P}^{1}$ of $F_{2}(x)$ correspond to semi-stable decomposable bundles, i.e., points of $\mathfrak{D}$. (Furthermore, the bundles corresponding to different points in $h_{\ell, x}^{-1}\left(F_{2}(x)\right)$ are not isomorphic to each other.) It is not clear why one would want to throw away this open dense subset of the Hecke fiber. More examples of this nature are given below.

Example 3.3. Let $\mathcal{M}=\mathcal{O}_{X} \oplus \mathcal{O}_{X}(x)$. Then, as explained in [7], there are two points in the fiber $h_{\ell, x}^{-1}(\mathcal{M})$, corresponding to $\mathcal{M}_{1}^{\prime}=\mathcal{O}_{X} \oplus \mathcal{O}_{X}$ and $\mathcal{M}_{2}^{\prime}=\mathcal{O}_{X}(-x) \oplus \mathcal{O}_{X}(x)$, and each point in the complement (which is isomorphic to $\mathbb{C}^{\times}$) corresponds to the indecomposable bundle $F_{2}$. Thus, we see that an open dense subset of the fiber $h_{\ell, x}^{-1}(\mathcal{M})$ of the Hecke correspondence over a decomposable rank two bundle $\mathcal{M}$ (corresponding to a point in $\mathfrak{D}$ ) consists of indecomposable bundles (corresponding to points of $\mathfrak{U}$ ).

Now, if we were to treat $\mathcal{M}_{1}^{\prime}=\mathcal{O}_{X} \oplus \mathcal{O}_{X}$ and $\mathcal{M}_{2}^{\prime}=\mathcal{O}_{X}(-x) \oplus \mathcal{O}_{X}(x)$ as belonging to a different connected component of $\mathrm{Bun}_{\mathrm{GL}_{2}}$ than $F_{2}$, then what to make of the integral (3.4)? It would seemingly break into the sum of two points and an integral over their complement. That would be fine in the case of a curve over $\mathbb{F}_{q}$ : we would simply obtain the formula

$$
\left(H_{1, x} \cdot f\right)(\mathcal{M})=f\left(\mathcal{M}_{1}^{\prime}\right)+f\left(\mathcal{M}_{2}^{\prime}\right)+(q-1) f\left(F_{2}\right),
$$

with the factor of $(q-1)$ being the number of points of $\mathbb{P}^{1}$ without two points (see $[1,45]$ ). But over complex numbers we have to integrate over $\mathbb{C}^{\times}$. We would therefore have to somehow combine summation over two points and integration over their complement. As in another example of this nature that we considered above, it is not clear that there exists an integration measure that would achieve this in a consistent and meaningful fashion.

Example 3.4. Similarly, if $\mathcal{M}=\mathcal{L}_{1} \oplus \mathcal{L}_{2}$, where $\mathcal{L}_{1}$ and $\mathcal{L}_{2}$ are non-isomorphic line bundles of degree 0 , then the fiber of the Hecke correspondence $h_{\ell, x}^{-1}(\mathcal{M})$ over $\mathcal{M}$ has two points corresponding $\mathcal{M}_{1}^{\prime}=\mathcal{L}_{1}(-x) \oplus \mathcal{L}_{2}$ and $\mathcal{M}_{2}^{\prime}=\mathcal{L}_{1} \oplus \mathcal{L}_{2}(-x)$, and every point in the complement of these two points (which is isomorphic to $\mathbb{C}^{\times}$) corresponds to the indecomposable vector bundle $F_{2}(x) \otimes \mathcal{L}(-x)$, where $\mathcal{L}^{\otimes 2} \simeq \mathcal{L}_{1} \otimes \mathcal{L}_{2}$. Again, we see that an open dense subset of the fiber $h_{\ell, x}^{-1}(\mathcal{M})$ of the Hecke correspondence over a decomposable rank two bundle $\mathcal{M}$ (corresponding to a point in $\mathfrak{D})$ consists of indecomposable bundles (corresponding to points of $\mathfrak{U}$ ). As in the previous example, it is not clear how to integrate over $h_{\ell, x}^{-1}(\mathcal{M})$.

Incidentally, Example 3.4 shows that for any $x \in X$ and any pair of degree zero line bundles $\mathcal{L}, \mathcal{L}_{1}$ on $X$, there is a continuous family of rank two vector bundles on $X$ over an affine line $\mathbb{A}^{1}$ that are isomorphic to $F_{2}(x) \otimes \mathcal{L}$ away from $0 \in \mathbb{A}^{1}$ and to $\mathcal{L}_{1}(x) \oplus\left(\mathcal{L}^{\otimes 2} \otimes \mathcal{L}_{1}^{-1}\right)$ at the point $0 \in \mathbb{A}^{1}$. Likewise, Example 3.3 shows that there exist continuous families of rank two vector bundles on $X$ over an affine line $\mathbb{A}^{1}$ that are isomorphic to $F_{2}$ away from $0 \in \mathbb{A}^{1}$ and to $\mathcal{O}_{X} \oplus \mathcal{O}_{X}$ or to $\mathcal{O}_{X}(-x) \oplus \mathcal{O}_{X}(x)$ at the point $0 \in \mathbb{A}^{1}$.

These examples illustrate the intricate (non-Hausdorff) topology of $\mathrm{Bun}_{\mathrm{GL}_{2}}$ of an elliptic curve; in particular, the fact that the substacks $D$ and $U$ of decomposable and indecomposable bundles are glued together in a highly non-trivial fashion.

To summarize: in [42] Langlands defines his versions of Hecke operators in the case of $\mathrm{GL}_{2}$ and an elliptic curve $X$ in an ad hoc fashion, without discussing the pertinent measures of integration from the first principles. As far as I understand, his definition is based on two assumptions: (i) the statement that the fibers of the Hecke correspondence are finite, see the 
quote from [42] at the beginning of this subsection; (ii) postulating that the Hecke operators should act on $L^{2}(\mathfrak{D}) \oplus L^{2}(\mathfrak{U})$ preserving each of the two direct summands. In this section, I have outlined the issues with these assumptions. ${ }^{8}$

\section{An alternative proposal}

There is however another approach to the analytic theory of automorphic functions for complex curves, proposed in a joint work with Pavel Etingof and David Kazhdan [16]. In this section I outline this approach.

\subsection{A toy model}

It is instructive to consider first a toy model for the questions we have been discussing. Over $\mathbb{F}_{q}$, there is a well-understood finite-dimensional analogue of the spherical Hecke algebra of $G\left(\mathbb{F}_{q}((t))\right)$; namely, the Hecke algebra $\mathcal{H}_{q}(G)$ of $B\left(\mathbb{F}_{q}\right)$ bi-invariant $\mathbb{C}$-valued functions on the group $G\left(\mathbb{F}_{q}\right)$, where $B$ is a Borel subgroup of a simple algebraic group $G$.

As a vector space, this algebra has a basis labeled by the characteristic functions $c_{w}$ of the Bruhat-Schubert cells $B\left(\mathbb{F}_{q}\right) w B\left(\mathbb{F}_{q}\right)$, where $w$ runs over the Weyl group of $G$. The convolution product on $\mathcal{H}_{q}(G)$ is defined using the constant measure $\mu_{q}$ on the finite group $G\left(\mathbb{F}_{q}\right)$ normalized so that the measure of $B\left(\mathbb{F}_{q}\right)$ is equal to 1 . Then the function $c_{1}$ is a unit element of $\mathcal{H}_{q}(G)$.

It is convenient to describe the convolution product on $\mathcal{H}_{q}(G)$ as follows: identify the $B$ biinvariant functions on $G$ with $B$-invariant functions on $G / B$ and then with $G$-invariant functions on $(G / B) \times(G / B)$ (with respect to the diagonal action). Given two $G$-invariant functions $f_{1}$ and $f_{2}$ on $(G / B) \times(G / B)$, we define their convolution product by the formula

$$
\left(f_{1} \star f_{2}\right)(x, y)=\int_{G / B} f_{1}(x, z) f_{2}(z, y) \mathrm{d} z .
$$

Under this convolution product, the algebra $\mathcal{H}_{q}(G)$ is generated by the functions $c_{s_{i}}$, where the $s_{i}$ are the simple reflections in $W$. They satisfy the well-known relations.

Observe also that the algebra $\mathcal{H}_{q}(G)$ naturally acts on the space $\mathbb{C}\left[G\left(\mathbb{F}_{q}\right) / B\left(\mathbb{F}_{q}\right)\right]$ of $\mathbb{C}$-valued functions on $G\left(\mathbb{F}_{q}\right) / B\left(\mathbb{F}_{q}\right)$. It acts on the right and commutes with the natural left action of $G\left(\mathbb{F}_{q}\right)$. Unlike the spherical Hecke algebra, $\mathcal{H}_{q}(G)$ is non-commutative. Nevertheless, we can use the decomposition of the space $\mathbb{C}\left[G\left(\mathbb{F}_{q}\right) / B\left(\mathbb{F}_{q}\right)\right]$ into irreducible representations of $\mathcal{H}_{q}(G)$ to describe it as direct sum of irreducible representations of $G\left(\mathbb{F}_{q}\right)$.

Now suppose that we wish to generalize this construction to the complex case. Thus, we consider the group $G(\mathbb{C})$, its Borel subgroup $B(\mathbb{C})$, and the quotient $G(\mathbb{C}) / B(\mathbb{C})$, which is the set of $\mathbb{C}$-points of the flag variety $G / B$ over $\mathbb{C}$. A naive analogue of $\mathcal{H}_{q}(G)$ would be the space $\mathcal{H}_{\mathbb{C}}(G)$ of $B(\mathbb{C})$ bi-invariant functions on $G(\mathbb{C})$. Therefore we have the following analogues of the questions that we discussed above in the case of the spherical Hecke algebra: Is it possible to define a measure of integration on $G(\mathbb{C})$ that gives rise to a meaningful convolution product

\footnotetext{
${ }^{8}$ Added in September 2019: If Bun $_{G}$ contains an open dense substack of stable bundles, it is possible to define analogues of Hecke operators acting on compactly supported sections of the line bundle of half-densities on this substack (rather than functions), following the construction of A. Braverman and D. Kazhdan [8] in the nonarchimedian case. The details will appear in [17]. However, this construction cannot be applied in the case of elliptic curves because there is no such open dense substack in $\operatorname{Bun}_{G}$ in this case (unless we add some extra structures to $G$-bundles, such as parabolic structures).

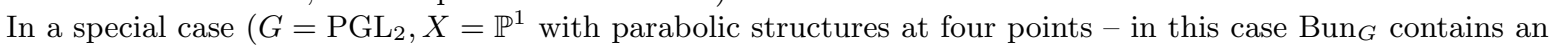
open dense substack of stable bundles), explicit formulas for the Hecke operators were proposed by M. Kontsevich [39, Section 2.4].
} 
on $\mathcal{H}_{\mathbb{C}}(G)$ ? Is it possible to use the resulting algebra to decompose the space of $L^{2}$ functions on $G(\mathbb{C}) / B(\mathbb{C})$ ?

For example, consider the case of $G=\mathrm{SL}_{2}$. Then $G / B=\mathbb{P}^{1}$. The Hecke algebra $\mathcal{H}_{q}\left(\mathrm{SL}_{2}\right)$ has a basis consisting of two elements, $c_{1}$ and $c_{s}$, which (in its realization as $G$-invariant functions on $(G / B) \times(G / B)$ explained above) correspond to the characteristic functions of the two $\mathrm{SL}_{2}$-orbits in $\mathbb{P}^{1} \times \mathbb{P}^{1}$ : the diagonal and its complement, respectively. Applying formula (4.1), we obtain that

$$
\begin{aligned}
& c_{1} \star c_{1}=c_{1}, \quad c_{1} \star c_{s}=c_{s}, \\
& c_{s} \star c_{s}=q c_{1}+(q-1) c_{s} .
\end{aligned}
$$

The two formulas in (4.2) follow from the fact that for each $x$ and $y$, in formula (4.1) there is either a unique value of $z$ for which the integrand is non-zero, or no such values. The coefficients in formula (4.3) have the following meaning: $q=\mu_{q}\left(\mathbb{A}^{1}\right), q-1=\mu_{q}\left(\mathbb{A}^{1} \backslash 0\right)$.

Now, if we try to adopt this to the case of $\mathbb{P}^{1}$ over $\mathbb{C}$, we quickly run into trouble. Indeed, if we want $c_{1}$ to be the unit element, we want to keep the two formulas in (4.2). But in order to reproduce the second formula in (4.2), we need a measure $d z$ on $\mathbb{C P}^{1}$ that would give us $\int \chi_{u} \mathrm{~d} z=1$ for every point $u \in \mathbb{C P}^{1}$, where $\chi_{u}$ is the characteristic function of $u$. However, then the integral of this measure over the affine line inside $\mathbb{C P}^{1}$ would diverge, rendering the convolution product $c_{s} \star c_{s}$ meaningless.

Likewise, we run into trouble if we attempt to define an action of $\mathcal{H}_{\mathbb{C}}(G)$ on the space of functions on $G(\mathbb{C}) / B(\mathbb{C})$. Thus, we see that the questions we asked above do not have satisfactory answers, and the reasons for that are similar to those we discussed in the previous section, concerning the spherical Hecke algebra and the possibility of defining an action of Hecke operators on functions on $\operatorname{Bun}_{G}$.

However, there are two natural variations of these questions that do have satisfactory answers. The first possibility is to consider a categorical version of the Hecke algebra, i.e., instead of the space of $B$-invariant functions, the category $D(G / B)^{B}$-mod of $B$-equivariant $D$-modules on $G / B$. According to a theorem of Beilinson and Bernstein [5], we have an exact functor of global sections (as $\mathcal{O}$-modules) from this category to the category of modules over the Lie algebra $\mathfrak{g}$ of $G$, which is an equivalence with the category of those $\mathfrak{g}$-module which have a fixed character of the center of $U(\mathfrak{g})$ (the character of the trivial representation of $\mathfrak{g}$ ). This is the category that appears in the Kazhdan-Lusztig theory, which gives rise, among other things, to character formulas for irreducible $\mathfrak{g}$-modules from the category $\mathcal{O}$. Furthermore, instead of the convolution product on functions, we now have convolution functors on a derived version of $D(G / B)^{B}$-mod. This is the categorical Hecke algebra which has many applications. For example, Beilinson and Bernstein have defined a categorical action of this category on the derived category of the category $\mathcal{O}$ (which may be viewed as the category of ( $\mathfrak{g}, B)$ Harish-Chandra modules). This is a special case of a rich theory.

Note that a closely related category of perverse sheaves may also be defined over $\mathbb{F}_{q}$. Taking the traces of the Frobenius on the stalks of those sheaves, we obtain the elements of the original Hecke algebra $\mathcal{H}_{q}(G)$. This operation transforms convolution product of sheaves into convolution product of functions. Thus, we see many parallels with the geometric Langlands Program (for more on this, see [22, Section 1.3.3]). In particular, the spherical Hecke algebra has a categorical analogue, for which a categorical version of the Satake isomorphism has been proved [30, 46, 47]. In other words, the path of categorification of the Hecke algebra $\mathcal{H}_{q}(G)$ is parallel to the path taken in the geometric Langlands theory.

But there is also a second option: We can define a Hilbert space $L^{2}(G(\mathbb{C}) / B(\mathbb{C})$ ) as the completion of the space of half-densities on $G(\mathbb{C}) / B(\mathbb{C})$ with respect to the natural Hermitian inner product (this has a generalization corresponding to twisting by line bundles on $G / B$ as well as certain "imaginary powers" thereof). However, instead of defining an action of a Hecke algebra on this Hilbert space, one then uses a substitute: differential operators on $G / B$. 
The Lie algebra $\mathfrak{g}$ acts on $L^{2}(G(\mathbb{C}) / B(\mathbb{C}))$ by holomorphic vector fields, and we have a commuting action of another copy of $\mathfrak{g}$ by anti-holomorphic vector fields. Therefore, the tensor product of two copies of the center of $U(\mathfrak{g})$ acts by mutually commuting differential operators. ${ }^{9}$ As we mentioned above, both holomorphic and anti-holomorphic ones act according to the central character of the trivial representation. However, the center of $U\left(\mathfrak{g}_{c}\right)$, where $\mathfrak{g}_{c}$ is a compact form of the Lie algebra $\mathfrak{g}$, also acts on $L^{2}(G(\mathbb{C}) / B(\mathbb{C}))$ by commuting differential operators, and this action is non-trivial. It includes the Laplace operator, which corresponds to the Casimir element of $U\left(\mathfrak{g}_{c}\right)$.

We then ask what are the eigenfunctions and eigenvalues of these commuting differential operators. This question has a meaningful answer. Indeed, using the isomorphism $G / B \simeq G_{c} / T_{c}$, where $T_{c}$ is a maximal torus of the compact form of $G$, and the Peter-Weyl theorem, we obtain that $L^{2}(G(\mathbb{C}) / B(\mathbb{C}))$ can be decomposed as a direct sum of irreducible finite-dimensional representations of $\mathfrak{g}_{c}$ which can be exponentiated to the group $G_{c}$ of adjoint type, each irreducible representation $V$ appearing with multiplicity equal to the dimension of the weight 0 subspace $V(0)$ of $V$. Therefore the combined action of the center of $U\left(\mathfrak{g}_{c}\right)$ and the Cartan subalgebra $\mathfrak{t}_{c}$ of $T_{c}$ (acting by vector fields) has as eigenspaces various weight components of various irreducible representations $V$ of $\mathfrak{g}_{c}$ tensored with $V(0)$. All of these eigenspaces are finite-dimensional.

For instance, for $G=\mathrm{SL}_{2}$ every eigenspace is one-dimensional, and so we find that these differential operators have simple spectrum. In fact, suitably normalized joint eigenfunctions of the center of $U\left(\mathfrak{g}_{c}\right)$ and $\mathfrak{t}_{c}$ are in this case the standard spherical harmonics (note that in this case $\left.G(\mathbb{C}) / B(\mathbb{C}) \simeq S^{2}\right)$.

This discussion suggests we may be able to build a meaningful analytic theory of automorphic forms on $\operatorname{Bun}_{G}$ if, rather than looking for the eigenfunctions of Hecke operators (whose existence is questionable in the non-abelian case, as we have seen), we look for the eigenfunctions of a commutative algebra of global differential operators on $\mathrm{Bun}_{G}$. It turns out that we are in luck: there exists a large commutative algebra of differential operators acting on the line bundle of half-densities on $\operatorname{Bun}_{G}$.

Remark 4.1. The above discussion dovetails nicely with the intuition that comes from the theory of automorphic functions for a reductive group $G$ over a number field $F$. Such a field has non-archimedian as well as archimedian completions. The representation theories of the corresponding groups, such as $G\left(\mathbb{Q}_{p}\right)$ and $G(\mathbb{C})$, are known to follow different paths: for the former we have, in the unramified case, the spherical Hecke algebra and the Satake isomorphism. For the latter, instead of a spherical Hecke algebra one usually considers the center of $U(\mathfrak{g})$ (or, more generally, the convolution algebra of distributions on $G(\mathbb{C})$ supported on its compact subgroup $K$, see [38]).

Now let's replace a number field $F$ by a field of the form $F(X)$, where $X$ is a curve over $F$. Then instead of the local fields $\mathbb{Q}_{p}$ we would have fields such as $\mathbb{Q}_{p}((t))$, and instead of $\mathbb{C}$ we would have $\mathbb{C}((t))$. In the former case we would have to consider the group $G\left(\mathbb{Q}_{p}((t))\right)$ and in the latter case, the group $G(\mathbb{C}((t)))$. For $G\left(\mathbb{Q}_{p}((t))\right)$ there are meaningful analogues of the spherical Hecke algebra and the corresponding Satake isomorphism. They have been studied, in particular, in $[8,9,35,36,37]$. But in the case of $G(\mathbb{C}((t)))$, just as in the case of a number field $F$ discussed above, it seems more prudent to consider the center of $U(\mathfrak{g}((t)))$ instead. As we show in the rest of this section, this approach leads to a rich and meaningful theory. Indeed, if we take the so-called critical central extension of $\mathfrak{g}((t))$, then the corresponding completed enveloping algebra does contain a large center, as shown in [18] (see also [21, 22]). This center gives rise to a large algebra of global commuting differential operators on $\mathrm{Bun}_{G}$.

\footnotetext{
${ }^{9}$ The referee drew my attention to the fact that this is essentially the classical Gelfand-Naimark construction of the principal series representations of $G(\mathbb{C})$ and the corresponding Harish-Chandra bimodules.
} 


\subsection{Global differential operators on Bun $_{G}$}

Let us assume for simplicity that $G$ is a connected, simply-connected, simple algebraic group over $\mathbb{C}$. In [6], Beilinson and Drinfeld have described the algebra $D_{G}$ of global holomorphic differential operators on $\operatorname{Bun}_{G}$ acting on the square root $K^{1 / 2}$ of a canonical line bundle (which exists for any reductive $G$ and is unique under our assumptions). They have proved that $D_{G}$ is commutative and is isomorphic to the algebra of functions on the space $\mathrm{Op}_{L} G(X)$ of ${ }^{L} G$-opers on $X$. For a survey of this construction and the definition of $\mathrm{Op}_{L_{G}}(X)$, see, e.g., [23, Sections 8 and 9]. Under the above assumptions on $G$, the space $\mathrm{Op}_{L_{G}}(X)$ may be identified with the space of all holomorphic connections on a particular holomorphic ${ }^{L} G$-bundle $\mathcal{F}_{0}$ on $X$. In particular, it is an affine space of dimension equal to $\operatorname{dim} \operatorname{Bun}_{L} G$.

The construction of these global differential operators is similar to the construction outlined in Section 4.1 above. Namely, they are obtained in [6] from the central elements of the completed enveloping algebra of the affine Kac-Moody algebra $\widehat{\mathfrak{g}}$ at the critical level, using the realization of $\operatorname{Bun}_{G}$ as a double quotient of the formal loop group $G(\mathbb{C}((t)))$ and the Beilinson-Bernstein type localization functor. The critical level of $\widehat{\mathfrak{g}}$ corresponds to the square root of the canonical line bundle on $\operatorname{Bun}_{G}$. A theorem of Feigin and myself [18] (see also [21, 22]) identifies the center of this enveloping algebra with the algebra of functions on the space of ${ }^{L} G$-opers on the formal punctured disc. This is a local statement that Beilinson and Drinfeld use in the proof of their theorem.

Now, we can use the same method to construct the algebra $\bar{D}_{G}$ of global anti-holomorphic differential operators on $\operatorname{Bun}_{G}$ acting on the square root $\bar{K}^{1 / 2}$ of the anti-canonical line bundle. The theorem of Beilinson and Drinfeld implies that $\bar{D}_{G}$ is isomorphic to the algebra of functions on the complex conjugate space to the space of opers, which we denote by $\overline{\mathrm{Op}}_{L}(X)$. Under the above assumptions on $G$, it can be identified with the space of all anti-holomorphic connections on the $G$-bundle $\overline{\mathcal{F}}_{0}$ that is the complex conjugate of the $G$-bundle $\mathcal{F}_{0}$. While $\mathcal{F}_{0}$ carries a holomorphic structure (i.e., a $(0,1)$-connection), $\overline{\mathcal{F}}_{0}$ carries a $(1,0)$-connection (which one could call an "anti-holomorphic structure" on $\left.\overline{\mathcal{F}}_{0}\right)$. Just as a $(1,0)$, i.e., holomorphic, connection on $\mathcal{F}_{0}$ completes its holomorphic structure to a flat connection, so does a $(0,1)$, i.e., anti-holomorphic, connection on $\overline{\mathcal{F}}_{0}$ complete its $(1,0)$-connection to a flat connection.

Both $\mathrm{Op}_{L}(X)$ and $\overline{\mathrm{Op}}_{L}(X)$ may be viewed as Lagrangian subspaces of the moduli stack of flat ${ }^{L} G$-bundles on $X$, and it turns out that it is their intersection that is relevant to the eigenfunctions of the global differential operators.

Indeed, we have a large commutative algebra $D_{G} \otimes \bar{D}_{G}$ of global differential operators on the line bundle $K^{1 / 2} \otimes \bar{K}^{1 / 2}$ of half-densities on $\operatorname{Bun}_{G}$. This algebra is isomorphic to the algebra of functions on $\mathrm{Op}_{L}(X) \times \overline{\mathrm{Op}}_{L}(X)$.

Let $\operatorname{Bun}_{G}^{\text {st }} \subset \operatorname{Bun}_{G}$ be the substack of stable $G$-bundles. Suppose that it is open and dense in $\operatorname{Bun}_{G}$ (this is equivalent to the genus of $X$ being greater than 1). We define the Hilbert space $L^{2}\left(\operatorname{Bun}_{G}\right)$ as the completion of the space $V$ of smooth compactly supported sections of $K^{1 / 2} \otimes \bar{K}^{1 / 2}$ over $\operatorname{Bun}_{G}^{\text {st }}$ with the standard Hermitian inner product.

The algebra $D_{G} \otimes \bar{D}_{G}$ preserves the space $V$ and is generated over $\mathbb{C}$ by those operators that are symmetric on $V$. These are unbounded operators on $L^{2}\left(\operatorname{Bun}_{G}\right)$, but we expect that a real form of the algebra $D_{G} \otimes \bar{D}_{G}$ has a canonical self-adjoint extension (this is explained in [16]). If so, then we get a nice set-up for the problem of finding joint eigenfunctions and eigenvalues of these operators. It is natural to call these eigenfunctions the automorphic forms on $\mathrm{Bun}_{G}$ (or $\operatorname{Bun}_{G}^{\mathrm{st}}$ ) for a complex algebraic curve. We expect that this can be generalized to an arbitrary connected reductive complex group $G$.

The joint eigenvalues of $D_{G} \otimes \bar{D}_{G}$ on $L^{2}\left(\operatorname{Bun}_{G}\right)$ correspond to points in $\mathrm{Op}_{L_{G}}(X) \times \overline{\mathrm{Op}}_{L}(X)$, i.e., pairs $(\chi, \rho)$, where $\chi \in \mathrm{Op}_{L} G(X)$ and $\rho \in \overline{\mathrm{Op}}_{L}(X)$. A joint eigenfunction corresponding 
to the pair $(\chi, \rho)$ satisfies the system of linear PDEs

$$
H_{i} \Psi=\chi\left(H_{i}\right) \Psi, \quad \bar{H}_{i} \Psi=\rho\left(\bar{H}_{i}\right) \Psi,
$$

where the $H_{i}$ (resp., the $\bar{H}_{i}$ ) are generators of $D_{G}$ (resp., $\bar{D}_{G}$ ), and $\chi$ (resp., $\rho$ ) is viewed as a homomorphism $D_{G} \rightarrow \mathbb{C}$ (resp., $\bar{D}_{G} \rightarrow \mathbb{C}$ ).

As far as I know, the system (4.4) was first considered by Teschner [50], in the case of $G=\mathrm{SL}_{2}$ (a similar idea was also proposed in [24]). Teschner did not consider (4.4) as a spectral problem in the sense of self-adjoint operators acting on a Hilbert space. Instead, he considered the problem of finding the set of real-analytic single-valued solutions $\Psi$ of the system (4.4) in which it is additionally assumed that $\rho=\bar{\chi}$ (note that this is not necessarily so if we do not have a self-adjointness property). He outlined in [50] how the solution to this problem can be related to those $\mathrm{PGL}_{2}$-opers (equivalently, projective connections) $\chi$ on $X$ that have monodromy taking values in the split real form $\mathrm{PGL}_{2}(\mathbb{R})$ of $\mathrm{PGL}_{2}(\mathbb{C}$ ) (up to conjugation by an element of $\mathrm{PGL}_{2}(\mathbb{C})$ ).

Projective connections with such monodromy have been described by Goldman [31]. If the genus of $X$ is greater than 1 , then among them there is a special one, corresponding to the uniformization of $X$. But there are many other ones as well, and they have been the subject of interest for many years. It is fascinating that they now show up in the context of the Langlands correspondence for complex curves.

In [16], we discuss the spectral problem associated to the system (4.4) for a general simplyconnected simple Lie group $G$. Though the $H_{i}$ and the $\bar{H}_{i}$ correspond to unbounded operators on the Hilbert space $L^{2}\left(\operatorname{Bun}_{G}\right)$, we conjecture that their linear combinations have canonical selfadjoint extensions. Furthermore, we conjecture (and prove in some cases) that the corresponding eigenvalues are the pairs $(\chi, \rho)$ such that $\rho=\bar{\chi}$ and $\chi$ is an ${ }^{L} G$-oper on $X$ whose monodromy takes values in the split real form of ${ }^{L} G$.

In the next subsection I will illustrate how these opers appear in the abelian case.

\subsection{The spectra of global differential operators for $G=G^{2} L_{1}$}

For simplicity, consider the elliptic curve $X=E_{\mathrm{i}}=C /(\mathbb{Z}+\mathbb{Z} \mathrm{i})$ discussed in Section 2.1. We identify the neutral component $\operatorname{Pic}^{0}(X)$ with $X$ using a reference point $p_{0}$, as in Section 2.1. Then the algebra $D_{\mathrm{GL}_{1}}$ (resp. $\bar{D}_{\mathrm{GL}_{1}}$ ) coincides with the algebra of constant holomorphic (resp. anti-holomorphic) differential operators on $X$ :

$$
D_{\mathrm{GL}_{1}}=\mathbb{C}\left[\partial_{z}\right], \quad \bar{D}_{\mathrm{GL}_{1}}=\mathbb{C}\left[\partial_{\bar{z}}\right] .
$$

The eigenfunctions of these operators are precisely the Fourier harmonics $f_{m, n}$ given by formula (2.4):

$$
f_{m, n}=\mathrm{e}^{2 \pi \mathrm{i} m x} \cdot \mathrm{e}^{2 \pi \mathrm{i} n y}, \quad m, n \in \mathbb{Z} .
$$

If we rewrite it in terms of $z$ and $\bar{z}$ :

$$
f_{m, n}=\mathrm{e}^{\pi z(n+\mathrm{i} m)} \cdot \mathrm{e}^{-\pi \bar{z}(n-\mathrm{i} m)}
$$

then we find that the eigenvalues of $\partial_{z}$ and $\partial_{\bar{z}}$ on $f_{m, n}$ are $\pi(n+\mathrm{i} m)$ and $-\pi(n-\mathrm{i} m)$ respectively. Let us recast these eigenvalues in terms of the corresponding $\mathrm{GL}_{1}$-opers.

By definition, a $\mathrm{GL}_{1}$-oper is a holomorphic connection on the trivial line bundle on $X$ (see [23, Section 4.5]). The space of such connections is canonically isomorphic to the space of holomorphic one-forms on $X$ which may be written as $-\lambda \mathrm{d} z$, where $\lambda \in \mathbb{C}$. An element of the 
space of $\mathrm{GL}_{1}$-opers may therefore be represented as a holomorphic connection on the trivial line bundle, which together with its $(0,1)$ part $\partial_{\bar{z}}$ yields the flat connection

$$
\nabla=d-\lambda \mathrm{d} z, \quad \lambda \in \mathbb{C} .
$$

Under the isomorphism $\operatorname{Spec} D_{\mathrm{GL}_{1}} \simeq \mathrm{Op}_{\mathrm{GL}_{1}}(X)$, the oper (4.5) corresponds to the eigenvalue $\lambda$ of $\partial_{z}$ (this is why we included the sign in (4.5)).

Likewise, an element of the complex conjugate space $\overline{\mathrm{Op}}_{\mathrm{GL}_{1}}(X)$ is an anti-holomorphic connection on the trivial line bundle, which together with its $(1,0)$ part $\partial_{z}$ yields the flat connection

$$
\bar{\nabla}=d-\mu \mathrm{d} \bar{z}, \quad \mu \in \mathbb{C} .
$$

Under the isomorphism $\operatorname{Spec} \bar{D}_{\mathrm{GL}_{1}} \simeq \overline{\mathrm{Op}}_{\mathrm{GL}_{1}}(X)$, the oper (4.6) corresponds to the eigenvalue $\mu$ of $\partial_{\bar{z}}$.

We have found above that the eigenvalues of $\partial_{z}$ and $\partial_{\bar{z}}$ on $L^{2}\left(\operatorname{Bun}_{\mathrm{GL}_{1}}\right)$ are $\pi(n+\mathrm{i} m)$ and $-\pi(n-\mathrm{i} m)$, respectively, where $m, n \in \mathbb{Z}$. The following lemma, which is proved by a direct computation, links them to $\mathrm{GL}_{1}$-opers with monodromy in $\mathrm{GL}_{1}(\mathbb{R})$.

Lemma 4.2. The connection (4.5) (resp. (4.6)) on the trivial line bundle on $E_{\mathrm{i}}=\mathbb{C}(\mathbb{Z}+\mathbb{Z} \mathrm{i})$ has monodromy taking values in the split real form $\mathbb{R}^{\times} \subset \mathbb{C}^{\times}$if and only if $\lambda=\pi(n+\mathrm{im})$ (resp. $\mu=-\pi(n-\mathrm{i} m))$, where $m, n \in \mathbb{Z}$.

This lemma generalizes in a straightforward fashion to arbitrary curves and arbitrary abelian groups. Namely, the harmonics $\mathrm{e}^{2 \pi \mathrm{i} \varphi_{\gamma}}, \gamma \in H^{1}\left(X, \Lambda^{*}(T)\right)$, introduced in Section 2.5 are the eigenfunctions of the global differential operators on $\operatorname{Bun}_{T}^{0}(X)$. The ${ }^{L} T$-oper on $X$ encoding the eigenvalues of the holomorphic differential operators is the holomorphic connection on the trivial ${ }^{L} T$-bundle on $X$

$$
\nabla_{\gamma}^{\mathrm{hol}}=d-2 \pi \mathrm{i} \omega_{\gamma}
$$

(compare with formula (2.25)). One can show that its monodromy representation takes values in the split real form of ${ }^{{ }} T$, and conversely, these are all the ${ }^{L} T$-opers on $X$ that have real monodromy. Thus, the conjectural description of the spectra of global differential operators on $\operatorname{Bun}_{G}$ in terms of opers with split real monodromy (see the end of Section 4.2) holds in the abelian case.

Recall that in the abelian case we also have well-defined Hecke operators. It is interesting to note that they commute with the global differential operators and share the same eigenfunctions. Furthermore, the eigenvalues of the Hecke operators may be expressed in terms of the eigenvalues of the global differential operators.

For non-abelian $G$, we consider global differential operators as substitutes for Hecke operators. We expect that their eigenvalues are given by the ${ }^{L} G$-opers satisfying a special condition: namely, their monodromy representation $\pi_{1}\left(X, p_{0}\right) \rightarrow{ }^{L} G$ takes values in the split real form of ${ }^{L} G$. It is

natural to view these homomorphisms as the Langlands parameters of the automorphic forms for curves over $\mathbb{C}$. The details will appear in [16].

\section{Acknowledgments}

The first version of this paper was based on the notes of my talk at the 6th Abel Conference, University of Minnesota, November 2018. I thank Roberto Alvarenga, Julia Gordon, Ivan Fesenko, David Kazhdan, and Raven Waller for valuable discussions. 


\section{References}

[1] Alvarenga R., Hall algebra and graphs of Hecke operators for elliptic curves, arXiv:1805.00567.

[2] Arinkin D., Gaitsgory D., Singular support of coherent sheaves and the geometric Langlands conjecture, Selecta Math. (N.S.) 21 (2015), 1-199, arXiv:1201.6343.

[3] Atiyah M.F., Vector bundles over an elliptic curve, Proc. London Math. Soc. 7 (1957), 414-452.

[4] Atiyah M.F., Bott R., The Yang-Mills equations over Riemann surfaces, Philos. Trans. Roy. Soc. London Ser. A 308 (1983), 523-615.

[5] Beilinson A., Bernstein J., A proof of Jantzen conjectures, in I.M. Gel'fand Seminar, Adv. Soviet Math., Vol. 16, Amer. Math. Soc., Providence, RI, 1993, 1-50.

[6] Beilinson A., Drinfeld V., Quantization of Hitchin's integrable system and Hecke eigensheaves, available at http://math.uchicago. edu/ drinfeld/langlands/QuantizationHitchin.pdf.

[7] Boozer D., Moduli spaces of Hecke modifications for rational and elliptic curves, arXiv:1805.11184.

[8] Braverman A., Kazhdan D., Some examples of Hecke algebras for two-dimensional local fields, Nagoya Math. J. 184 (2006), 57-84, arXiv:math.RT/0510538.

[9] Braverman A., Kazhdan D., The spherical Hecke algebra for affine Kac-Moody groups I, Ann. of Math. 174 (2011), 1603-1642, arXiv:0809.1461.

[10] Casselman W., Cely J.E., Hales T., The spherical Hecke algebra, partition functions, and motivic integration, Trans. Amer. Math. Soc. 371 (2019), 6169-6212, arXiv:1611.05773.

[11] Cluckers R., Loeser F., Constructible motivic functions and motivic integration, Invent. Math. 173 (2008), 23-121, arXiv:math.AG/0410203.

[12] Drinfeld V.G., Langlands' conjecture for GL(2) over functional fields, in Proceedings of the International Congress of Mathematicians (Helsinki, 1978), Acad. Sci. Fennica, Helsinki, 1980, 565-574.

[13] Drinfeld V.G., Two-dimensional $l$-adic representations of the fundamental group of a curve over a finite field and automorphic forms on GL(2), Amer. J. Math. 105 (1983), 85-114.

[14] Drinfeld V.G., Moduli varieties of F-sheaves, Funct. Anal. Appl. 21 (1987), 107-122.

[15] Drinfeld V.G., The proof of Petersson's conjecture for GL(2) over a global field of characteristic $p$, Funct. Anal. Appl. 22 (1988), 28-43.

[16] Etingof P., Frenkel E., Kazhdan D., An analytic version of the Langlands correspondence for complex curves, arXiv:1908.09677.

[17] Etingof P., Frenkel E., Kazhdan D., Hecke operators for curves over local fields, in preparation.

[18] Feigin B., Frenkel E., Affine Kac-Moody algebras at the critical level and Gelfand-Dikii algebras, Internat. J. Modern Phys. A 7 (1992), suppl. 1A, 197-215.

[19] Fesenko I., Analysis on arithmetic schemes. I, 2003, extra vol., 261-284.

[20] Fesenko I., Measure, integration and elements of harmonic analysis on generalized loop spaces, in Proceedings of the St. Petersburg Mathematical Society, Vol. XII, Amer. Math. Soc. Transl. Ser. 2, Vol. 219, Amer. Math. Soc., Providence, RI, 2006, 149-165.

[21] Frenkel E., Wakimoto modules, opers and the center at the critical level, Adv. Math. 195 (2005), 297-404, arXiv:math.QA/0210029.

[22] Frenkel E., Langlands correspondence for loop groups, Cambridge Studies in Advanced Mathematics, Vol. 103, Cambridge University Press, Cambridge, 2007.

[23] Frenkel E., Lectures on the Langlands program and conformal field theory, in Frontiers in Number Theory, Physics, and Geometry. II, Springer, Berlin, 2007, 387-533, arXiv:hep-th/0512172.

[24] Frenkel E., Gauge theory and Langlands duality, Talk given at MSRI, Berkeley, September 2014, available at https: //youtu.be/NQfeBeRKMiw?t=4190.

[25] Frenkel E., Gaitsgory D., Vilonen K., On the geometric Langlands conjecture, J. Amer. Math. Soc. 15 (2002), 367-417, arXiv:math.AG/0012255.

[26] Gaitsgory D., On a vanishing conjecture appearing in the geometric Langlands correspondence, Ann. of Math. 160 (2004), 617-682, arXiv:math.AG/0204081.

[27] Gaitsgory D., Outline of the proof of the geometric Langlands conjecture for $\mathrm{GL}_{2}$, Astérisque $\mathbf{3 7 0}$ (2015), 1-112, arXiv:1302.2506. 
[28] Gaitsgory D., Progrès récents dans la théorie de Langlands géométrique, Astérisque 390 (2017), exp. no. 1109, 139-168, arXiv:1606.09462.

[29] Gawędzki K., Lectures on conformal field theory, in Quantum Fields and Strings: a Course for Mathematicians, Vols. 1, 2 (Princeton, NJ, 1996/1997), Amer. Math. Soc., Providence, RI, 1999, 727-805.

[30] Ginzburg V., Perverse sheaves on a loop group and Langlands duality, arXiv:alg-geom/9511007.

[31] Goldman W.M., Projective structures with Fuchsian holonomy, J. Differential Geom. 25 (1987), $297-326$.

[32] Gordon J., Motivic Haar measure on reductive groups, Canad. J. Math. 58 (2006), 93-114, arXiv:math.AG/0203106.

[33] Griffiths P., Harris J., Principles of algebraic geometry, Pure and Applied Mathematics, Wiley-Interscience, New York, 1978.

[34] Hrushovski E., Kazhdan D., Integration in valued fields, in Algebraic geometry and number theory, Progr. Math., Vol. 253, Birkhäuser Boston, Boston, MA, 2006, 261-405, arXiv:math.AG/0510133.

[35] Hrushovski E., Kazhdan D., The value ring of geometric motivic integration, and the Iwahori Hecke algebra of $\mathrm{SL}_{2}$, Geom. Funct. Anal. 17 (2008), 1924-1967, arXiv:math.AG/0609115.

[36] Kapranov M., Double affine Hecke algebras and 2-dimensional local fields, J. Amer. Math. Soc. 14 (2001), 239-262, arXiv:math.AG/9812021.

[37] Kim H.H., Lee K.H., Spherical Hecke algebras of $\mathrm{SL}_{2}$ over 2-dimensional local fields, Amer. J. Math. 126 (2004), 1381-1399.

[38] Knapp A.W., Vogan Jr. D.A., Cohomological induction and unitary representations, Princeton Mathematical Series, Vol. 45, Princeton University Press, Princeton, NJ, 1995.

[39] Kontsevich M., Notes on motives in finite characteristic, in Algebra, Arithmetic, and Geometry: in Honor of Yu.I. Manin, Vol. II, Progr. Math., Vol. 270, Birkhäuser Boston, Boston, MA, 2009, 213-247, arXiv:math.AG/0702206.

[40] Lafforgue L., Chtoucas de Drinfeld et correspondance de Langlands, Invent. Math. 147 (2002), 1-241.

[41] Langlands R.P., Problems in the theory of automorphic forms, in Lectures in Modern Analysis and Applications, III, Lecture Notes in Math., Vol. 170, Springer, Berlin, 1970, 18-61.

[42] Langlands R.P., On the analytical form of the geometric theory of automorphic forms, in Russian, available at http://publications.ias.edu/rpl/paper/2678.

[43] Laumon G., Correspondance de Langlands géométrique pour les corps de fonctions, Duke Math. J. 54 (1987), 309-359.

[44] Laumon G., Transformation de Fourier, constantes d'équations fonctionnelles et conjecture de Weil, Inst. Hautes Études Sci. Publ. Math. 65 (1987), 131-210.

[45] Lorscheid O., Automorphic forms for elliptic function fields, Math. Z. 272 (2012), 885-911, arXiv:1012.4825.

[46] Lusztig G., Singularities, character formulas, and a q-analog of weight multiplicities, Astérisque 101 (1983), 208-229.

[47] Mirković I., Vilonen K., Geometric Langlands duality and representations of algebraic groups over commutative rings, Ann. of Math. 166 (2007), 95-143, arXiv:math.RT/0401222.

[48] Morrow M., Integration on product spaces and $\mathrm{GL}_{n}$ of a valuation field over a local field, Commun. Number Theory Phys. 2 (2008), 563-592, arXiv:0712.2175.

[49] Morrow M., Integration on valuation fields over local fields, Tokyo J. Math. 33 (2010), 235-281, arXiv:0712.2172.

[50] Teschner J., Quantisation conditions of the quantum Hitchin system and the real geometric Langlands correspondence, arXiv:1707.07873.

[51] Waller R., Private communication, November 2018.

[52] Waller R., Measure and integration on non-locally compact spaces and representation theory, Ph.D. Thesis, University of Nottingham, 2019.

[53] Weil A., A 1940 letter of André Weil on analogy in mathematics (translated by Martin H. Krieger), Notices Amer. Math. Soc. 52 (2005), 334-341. 